\title{
Contribution of the basal forebrain to corticocortical network interactions
}

\author{
Peter Gombkoto $^{1,2} \cdot$ Matthew Gielow $^{1} \cdot$ Peter Varsanyi ${ }^{1} \cdot$ Candice Chavez $^{1} \cdot$ Laszlo Zaborszky $^{1}$
}

Received: 15 October 2020 / Accepted: 5 May 2021 / Published online: 22 May 2021

(c) The Author(s) 2021

\begin{abstract}
Basal forebrain (BF) cholinergic neurons provide the cerebral cortex with acetylcholine. Despite the long-established involvement of these cells in sensory processing, attention, and memory, the mechanisms by which cholinergic signaling regulates cognitive processes remain elusive. In this study, we recorded spiking and local field potential data simultaneously from several locations in the BF, and sites in the orbitofrontal and visual cortex in transgenic ChAT-Cre rats performing a visual discrimination task. We observed distinct differences in the fine spatial distributions of gamma coherence values between specific basalo-cortical and cortico-cortical sites that shifted across task phases. Additionally, cholinergic firing induced spatial changes in cortical gamma power, and optogenetic activation of BF increased coherence between specific cortico-cortical sites, suggesting that the cholinergic system contributes to selective modulation of cortico-cortical circuits. Furthermore, the results suggest that cells in specific BF locations are dynamically recruited across behavioral epochs to coordinate interregional cortical processes underlying cognition.
\end{abstract}

Keywords Basal forebrain · Orbitofrontal cortex $\cdot$ Visual association cortex $\cdot$ Gamma coherence $\cdot$ Optogenetics $\cdot$ Highdensity recordings

\section{Introduction}

The basal forebrain (BF) corticopetal projection system is the main source of acetylcholine (ACh) for all neocortical areas. $\mathrm{BF}$ areas rich in cholinergic neurons also contain GABAergic and glutamatergic corticopetal projection neurons, as well as various interneuron cell types (Gritti et al. 2003; Zaborszky and Gombkoto 2018). This anatomically complex system has been implicated in cortical activation, affect, attention, sensory coding, motivation, and memory. Lesions or blockade of $\mathrm{ACh}$ in the cortex results in impairments in perception (Minces et al. 2013), cognitive flexibility (Prado et al. 2017), executive function, and cortical plasticity (Conner et al. 2003, 2010; Ballinger et al. 2016). Evidence from tracing studies and lesions has suggested that the cholinergic projection system is organized

Laszlo Zaborszky

laszloz@newark.rutgers.edu

1 Center for Molecular and Behavioral Neuroscience, Rutgers University, 197 University Avenue, Newark, NJ 07102, USA

2 ETH Zurich Institute of Neuroinformatics, 8057 Zurich, Switzerland topographically: for example, posterior lesions of BF produce more damage to the cholinergic innervation of the auditory cortex compared to anterior regions (Chavez and Zaborszky 2017). Early functional data, however, have contributed to the view that the cholinergic signaling in the cortex is a slow, non-specific one, most likely acting through volume transmission (Hasselmo 2006; Sarter 2007; Parikh et al. 2007), and that the BF cholinergic projections are part of the "diffuse cortical projection systems" (Saper 1987). Recent anatomical studies paint a more complex picture, wherein the cholinergic projection to the neocortex is not diffuse, but instead is organized into cortical targetspecific groups of cholinergic neurons that receive specific combinations of inputs (Zaborszky et al. 2015; Gielow and Zaborszky 2017; Chavez and Zaborszky 2017). Moreover, cholinergic cells that project to the superficial or deep layers of the medial prefrontal cortex (mPFC) in mice are largely separated in the BF (Bloem et al. 2014). Also, new evidence from real-time amperometric recording indicates cholinergic signaling in attentional contexts that is rapid, phasic, transient, and probably synaptic (Sarter and Lustig, 2020). Based on the suggestion that the organization of projections from the BF may enable parallel modulation of multiple 


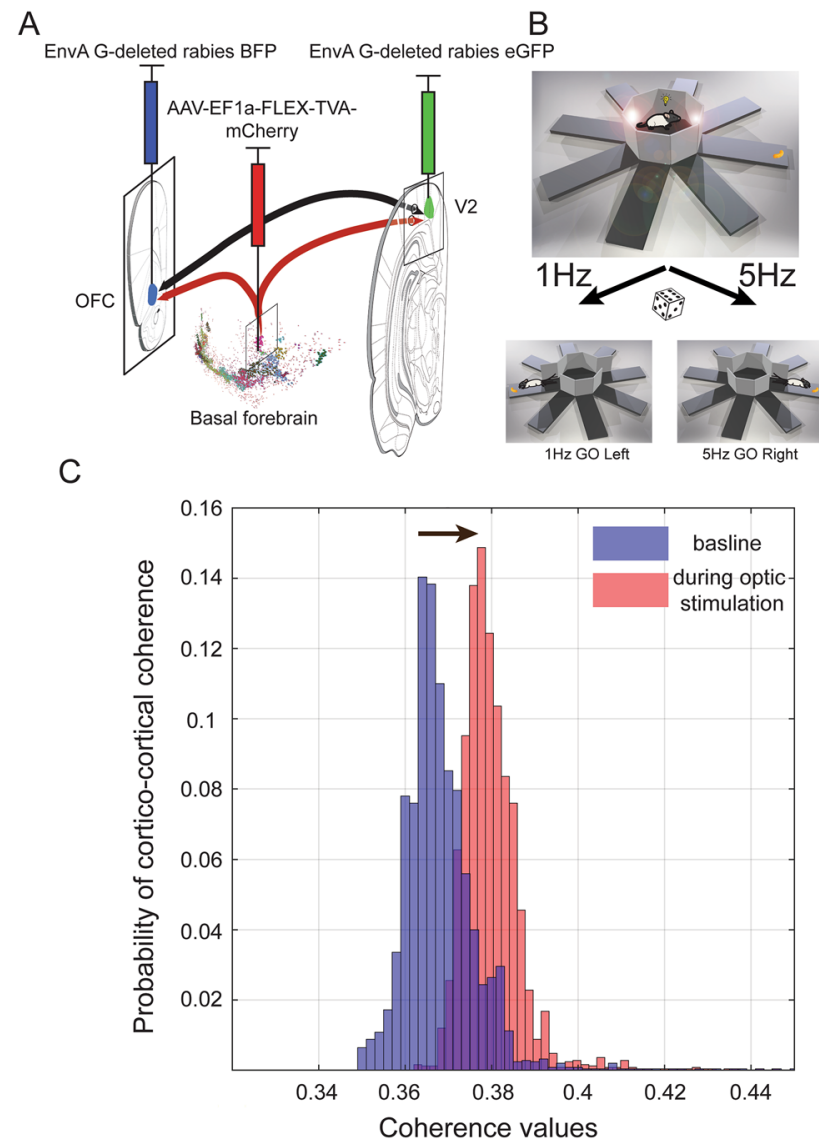

D

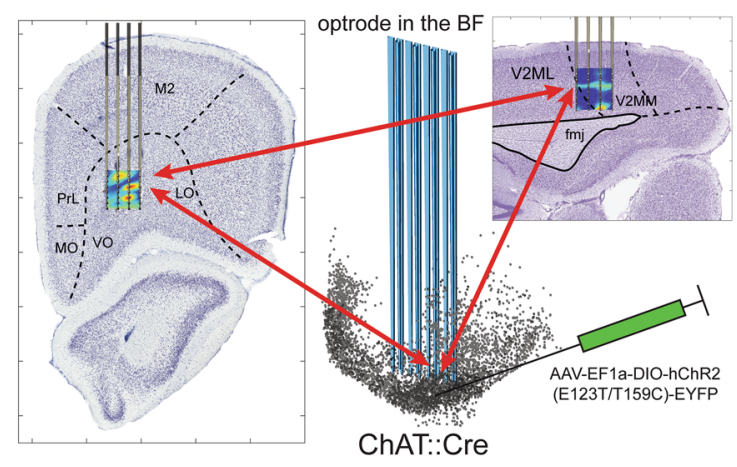

Fig. 1 Experimental paradigm. a To study cholinergic projections to V2 and $\mathrm{OFC}$, one animal received a helper virus in the ipsilateral $\mathrm{BF}$ as described (Gielow and Zaborszky 2017), followed by EnvA G-deleted rabies BFP injected in the OFC and EnvA G-deleted rabies eGFP into the V2 cortex. b Visual discrimination task. For the discrimination task, radial arm maze was used utilizing only 2 arms. The doors were closed until a flashing light cue occurs on both doors at two different frequencies $(1 \mathrm{~Hz}$ left, $5 \mathrm{~Hz}$ right) and then both doors opened. c Demonstration of the probability distribution of cortico-cortical coherence between OFC (Channel\#2) and V2 (Channel\#124). The blue histogram represents the coherence probability of the baseline. Red histogram represents the cortico-cortical coherence values during optic stimulation of cholinergic cells. Black arrow shows the shift of the cortico-cortical coherence by cholinergic activation. d Schematic position of silicon electrode array (blue lines with optic fibers attached at alternate shanks) within the cholinergic system (black dots in middle panel). Left: coronal section showing the position of silicon probes in ventral (VO) and lateral orbital (LO) cortex superimposed with local heat map of gamma coherence during decision making; Right: probes in V2 on a sagittal section superimposed with a heat map of local maxima of gamma coherence values during cue presentation. The red arrows represent the conceptualized functional loop between the structures groupings of interconnected yet nonadjacent cortical areas (Zaborszky et al., 2015), the aim of the current study is to elucidate the mechanism by which cortico-cortical interactions may be modulated by the BF.

The introduction of multi-electrode arrays (silicon probes) in awake, behaving rodents have begun to unravel the behavioural correlates of BF neurons, although the transmitter character of the recorded neurons was initially elusive (Zaborszky and Gombkoto 2018). For example, a tonic-firing subset of BF neurons burst transiently as an ensemble (Lin et al. 2006), and their synchronization phase was tightly associated with prefrontal (PFC) oscillation power in both the low $(<10 \mathrm{~Hz})$ and gamma range $(30-100 \mathrm{~Hz})$. The firing of these putative non-cholinergic neurons was correlated with the salience of reward- or punishment-predicting stimuli, irrespective of their sensory properties. Another recent study recorded spontaneous population activity in the BF and auditory cortex (Yague et al. 2017); however, their BF probe was not implanted in the region containing most of the auditory-projecting cholinergic neurons (Chavez and Zaborszky 2017). Based on the rhythmic firing of a large percentage (45.5\%) of neurons mainly at slow frequencies $(<6 \mathrm{~Hz})$, Yague et al. (2017) suggested that BF neurons slowly modulate cortical neurons. In contrast to these studies, the group led by Chiba and Nitz (Tingley et al. 2014, 2015, 2018), using a large dataset of BF neuronal activity ( $n=1428)$, suggested that the firing rate dynamics of populations of BF neurons oscillate in a nested fashion ("multiplexing") at different frequencies, including theta, beta, low and high gamma. These latter authors further suggest that this multiplexing maximizes information transfer from the $\mathrm{BF}$ to those cortical regions relating to working memory, reward encoding, motor states, and decision making.

Given the anatomical inhomogeneity of the BF, studies using single-unit recordings that do not systematically sample throughout the BF (Lin and Nicolelis 2008; Tingley et al. 2015) do not directly address the question of whether the BF modulates disparate cortical regions globally or in a selective fashion. Here, we use silicon probes in awake behaving rats (Fig. 1) to examine the coherence of the basalo-cortical and cortico-cortical networks (Fig. 1c-d), and the role of optogenetically stimulated cholinergic neurons in this process. With this, we are able to record and analyze electrophysiological datasets of meso- to large-scale networks that were previously described only in anatomical studies. Extracellular spikes and LFPs (local field potentials) were recorded simultaneously using 64-contact multi-shank probes chronically implanted in the BF; in addition, two 32-contact multi-shank neuronal probes were implanted in the orbitofrontal (OFC) and visual association cortex (V2) (Fig. 1d). Based on anatomical (Zaborszky 2002) and recent functional studies showing coordinated ACh release (TelesGrilo Ruivo et al. 2017), we also hypothesized that V2 and 
OFC areas, which are reciprocally interconnected (Reep et al. 1996), may be jointly modulated by the same BF neurons, including cholinergic cells.

\section{Materials and methods}

Viral tracing was used to reveal cholinergic projections from $\mathrm{BF}$ to their cortical (OFC, V2) targets. To compare neuronal activity between multiple cortical and BF subregions, we performed in vivo freely moving electrophysiological experiments in rats. Multi-electrode single-unit spike activity and LFPs were recorded simultaneously from different cortical layers of OFC, V2, and different compartments of the $\mathrm{BF}$ in three rats during successfully-learned visual cue discrimination. Supplementary Figure S1 shows an example recording during a single behavioral trial, displaying continuous wavelet transformation (CWT) of LFPs and population firing across all three brain regions, including acceleration of the animal in different behavioral epochs (Fig. S1g). The electrode array sampled across the entire BF, including the ventral pallidum (VP), substantia innominata (SI), horizontal limb of the diagonal band (HDB), and globus pallidus (GP), but excluding medial septum (MS) regions and the most posterior part of the BF.

\section{Animals}

Animals were treated in accordance with the National Research Council's "Guide for the Care and Use of Laboratory Animals" and with the approval of the Rutgers University Institutional Animal Care and Use Committee. ChAT::Cre rats expressing Cre under the ChAT promoter (Witten et al. 2011), a gift from Dr. Karl Deisseroth at Stanford University, were backcrossed with wild-type Long-Evans rats (Harlan). Three naive ChAT::Cre adults (5+ months of age) were used for the in vivo extracellular electrophysiology experiment; a fourth rat was used only to test the recording and optical stimulation system. Additionally, one animal was used for tracing cholinergic projections to V2 and OFC in ChAT::Cre rat. All rats were housed in cages of one to three animals on a 12-h-light/12-h-dark cycle.

\section{Viruses and surgeries}

Subjects were anesthetized with $1-4 \%$ isoflurane inhalation in $\mathrm{O} 2$. Rats received an intracranial injection of 2.64 $\mu \mathrm{L}$ virus (University of Pennsylvania: AAV-EF1a-DIOhChR2(E123T/T159C)-EYFP Serotype 5, titer $\geq 1 \times 1013$ $\mathrm{vg} / \mathrm{mL}$ ) using a Nanoject-II (Drummond Scientific) via micropipette across five locations covering the right $\mathrm{BF}$ (coordinates in $\mathrm{mm}$ relative to bregma, from pia, $+0.5 \mathrm{AP}$,
1.05 ML, 6 DV; 0.0 AP, 1.65 ML, 6.5DV; 0.87 AP, 2.3 ML, 5.2 and 6.5 DV; 1.7 AP, 3.4 ML, 5.6 DV). In order to study cholinergic projections to $\mathrm{V} 2$ and $\mathrm{OFC}$, one animal received helper virus (AAV-EF1a-FLEX-TVA-mCherry and AAVCA-FLEX-RG) in the ipsilateral BF as described (Gielow and Zaborszky 2017), followed by EnvA G-deleted rabies BFP injected in the OFC and EnvA G-deleted rabies eGFP into the V2 cortex; The coordinate for OFC was $4.2 \mathrm{AP} ; 2$ and 1.25 ML, DV 4.2, and for V2 -7.08 AP, 2 ML, 1 DV (Fig. 1a). Blue and red signals were enhanced (Fig. 2) with BFP (N0502-At647N-S FluoTag-X2, ATTO 647N-labeled), and RFP (N0401-SC3-S RFP FluoTag-Q, Sulfo-Cyanine 3-labeled) antibodies. Antibodies were diluted 1:500 in $0.1 \mathrm{M}$ PB (pH 7.4), 3\% normal goat serum and $0.1 \%$ triton. Sections were incubated for $3 \mathrm{~h}$ at room temperature, mounted on slides and cover slipped with DPX (VWR product code: $360294 \mathrm{H})$. Sections were incubated for $3 \mathrm{~h}$ at room temperature, mounted on slides and cover slipped with DPX (VWR product code: $360294 \mathrm{H})$. Forebrain sections ( $n=23,200 \mu \mathrm{m}$ series) were viewed by an upright confocal microscope (BX61WI, Olympus). The three channels used: GFP-excitation 488, emission filter 500-545; mCherryexcitation 559, emission filter 575-675; BFP-excitation 405; emission filter 425-475. Panel (d) in Fig. 2 is an image merged by Adobe Photoshop ${ }^{R}$ with no changes in the contrast or brightness.

\section{Behavioral training}

Behavioral training was conducted in a radial arm maze utilizing only 2 arms 180 degrees apart (Fig. 1). The central doors were closed until a flashing light cue (4 s duration) occurred on one of the doors at one of two different frequencies: $1 \mathrm{~Hz}$ indicating reward on the left arm, $5 \mathrm{~Hz}$ indicating reward on the right arm (Fig. 1b). Next, the two doors were opened simultaneously, and the reward (cheesy poof by Cheetos ${ }^{\circledR}$ ) was presented at the end of the correct corresponding arm. The reward was not visible to the rat from the gate because the gate was closed when the reward was delivered, in a $1 \mathrm{~cm}$ high and $2 \mathrm{~cm}$ diameter container at the end of the arm. After consumption of the reward, the rats freely returned to the start position at the center of the maze, and the gates were closed. The rats then waited for the next light cue for the location of the next reward. The cue light/reward location alternated sequentially until the rats got $90 \%$ correct, after which the trials were randomly intermixed. Each trial duration was verified manually based on accelerometer data and head position. The behavioral epochs were determined based on the location and acceleration of the rat and divided into 6 different time periods: center/resting, cue presentation (4 $\mathrm{s}$ duration), decision making (between door opening and initiation of arm approach), reward approach (during running 


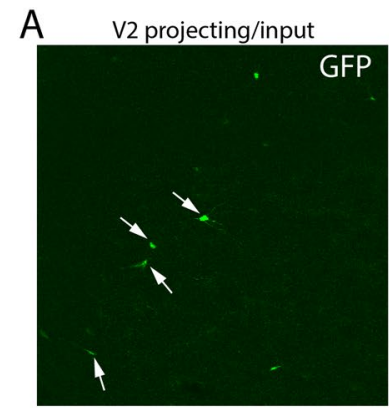

C

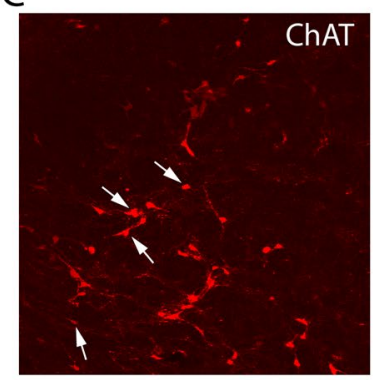

B

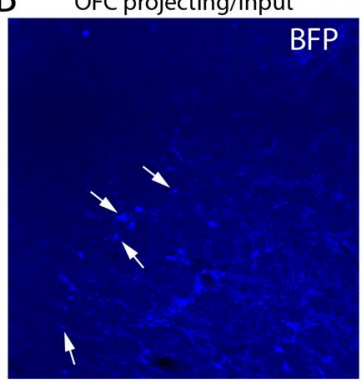

D

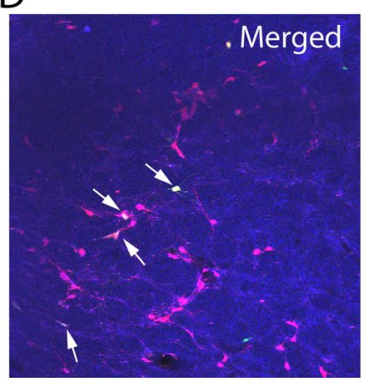

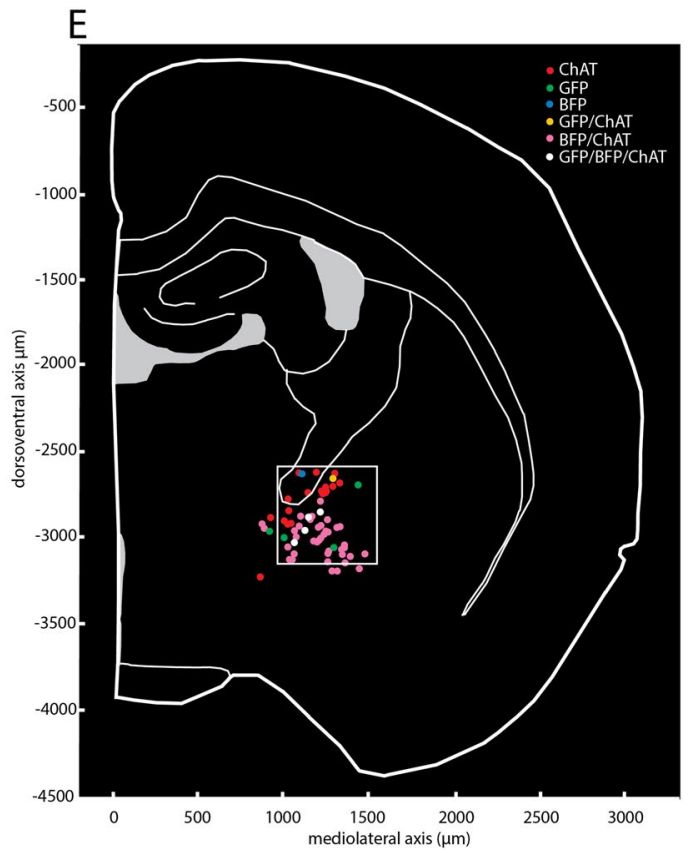

Fig. 2 Cholinergic (ChAT) projection neurons to V2 and OFC. a V2 projecting GFP labeled neurons, b BFP labeled neurons projecting to OFC, $\mathbf{c}$ mCherry-labeled cholinergic neurons, $\mathbf{d}$ merged images from $(\mathbf{a}-\mathbf{c})$. Arrows, point in each panel to triple-labeled neurons that is cholinergic and collateralized to $\mathrm{V} 2$ and $\mathrm{OFC}$, e schematic figure to show the distribution of differently labeled cells in this field of view. Explanation of various color-tagged cells is indicated in upper right. Please note that single green, or single blue cells represent input cells to cholinergic neurons projecting to $\mathrm{V} 2$ or OFC, respectively

puncturing large vasculature and were inserted into the brain. The visual cortex silicon electrode was positioned parallel to the median sagittal axis \{Bregma: from -6.12 to $-5.32 \mathrm{ML}: 2 \mathrm{~mm}$, maximum DV $1.4 \mathrm{~mm}$. The OFC electrode was positioned coronally \{Bregma: $4.2 \mathrm{~mm}$ ML: $1.2-2 \mathrm{~mm}$, DV: $3.5-4.3 \mathrm{~mm}$ \}. In total, 45 recording sessions were performed in three animals $(11,18$, and 16 sessions individually). A silicone gel (Dow Corning 3-4680 Krayden -DC4027868) was applied in the hole around the shanks to protect the cortical surface and prevent leakage of CSF. Prior to waking, subjects were given Buprenorphine $(0.04 \mathrm{mg} / \mathrm{kg}$, subcutaneous) and Meloxicam (1 mg/ $\mathrm{kg}$ subcutaneous) to prevent pain and inflammation. The micromanipulator (custom 3D-printed electrode microdrivers) and a 3D printed cap with copper mesh shield were cemented to the skull around the entire assembly to protect and electrically shield the electrodes while tightly holding the connectors.

\section{Recording procedures}

One week following implantation, rats were food-deprived and trained to run on a radial arm maze that they were previously acclimated to. Extracellular broadband $(0.33-5000 \mathrm{~Hz})$ signals were recorded simultaneously from all implanted 
probes. Recordings occurred daily for approximately one hour until recordings were no longer viable, indicating the endpoint. For the duration of the recording session, a digital head stage was connected to the 'probes' output connectors. For tracking the position of animals, two small LEDs mounted to the cap (front and rear) were recorded by a digital camera (1080p-30f/s). Video tracking was analyzed in real-time by LabVIEW software (National Instruments).

\section{Behavior control, data acquisition, and analysis}

The behavior hardware (motorized doors, digital camera, sensors from the shutter) and laser power supply were connected to a computer board (National Instruments \#USB6341) and controlled by LabVIEW and MATLAB (MathWorks, Natick, MA). Neurophysiological signals were sampled continuously at $20 \mathrm{kHz}$ on a 1024-channel digital acquisition system (http://www.hinstra.com/ready-solutions/ dedas) (Hinstra Instruments, Szeged, Hungary). The broadband signals were high-pass filtered $(0.8-5 \mathrm{kHz})$ offline for spike detection, and low pass filtered $(0-300 \mathrm{~Hz})$ and downsampled to $1250 \mathrm{~Hz}$ for local field potentials. For automatic spike sorting, we used Klusta, an open-source neurophysiological data analysis package in Python (Rossant et al. 2016). For manual refinement of sorting, Phy package (part of Klusta) was used.

\section{Laser light stimulation of neurons}

A blue laser (473 nm CrystaLaser CL-473-050—output power at the tip of optic fibers $5 \mathrm{~mW} \pm 0.5 \%$, measured by Thorlabs PM100D Optical Power and Energy Meter Console with S120C sensor controlled by analog input combined with a shutter) generated a $10 \mathrm{~ms}$ constant power stimulus train. The shutter was constructed from a disassembled hard drive, with the addition of a simple control circuit for the coil of the pivot arm and the spin of the disk. The shutter was built based on the concept of Maguire et al. (Maguire et al. 2004) but it was modified as follows: the spinning disk with a hole acted as the shutter (constantly spinning with a hole) which sequentially covered and uncovered the beam. The coil with the arm worked as a secondary "slow" shutter, which opened and closed the path of the output of the already-shuttered laser beam. In the off position of the arm, we could monitor the exact duration of the laser beam before we used it for stimulation, via a photodiode attached to the head of the arm. We used fiber optic rotary joint patch cables (Thorlabs \#FT200EMT). Cholinergic neurons were optogenetically tagged by laser stimulation $(10 \mathrm{~ms}$ train for $2 \mathrm{~s}, 5 \mathrm{~mW}$ ) at the end of each session (last five minutes of the session without any behavior). We tested each neuron with at least 100 stimuli. For each cell, we generated peri-light-stimulus time histograms (Fig. S3 third column: optostimulation) to analyze: the proportion of light-responsive neurons, defined as firing rate during light pulse $>2$ SDs above or below control firing rate $(2000 \mathrm{~ms}$ epoch before the stimulus).

\section{Behavior-specific single-unit activity (Fig. 3)}

Changes in ongoing firing rates during different behavioral epochs were analyzed by comparing firing rates during cue onset, decision making, and reward approach epochs vs. baseline using Wilcoxon rank-sum test (Fig. 3, Fig S2). For baseline recordings, we used spike trains from the behavioral epochs when the rats were in the center of the maze at rest.

\section{Coherence changes between behavioral epoch (Fig. 4)}

Using LFPs simultaneously recorded in multiple brain structures, we calculated the coherence between the structures in the low and high gamma bands. Continuous Wavelet Transformation (CWT) was used to obtain a time-frequency spectrogram of LFPs on all channels from BF, OFC, and V2 to assess behaviorally-related changes (Fig. S1 a, $b, c)$. The coherence values of behavioral epochs and during optic stimulations were normalized as a proportion of baseline coherence, which was measured at the beginning of each trial when the animals were located at the center start position of the maze, with the doors closed. Significant changes in coherence (averaged across all channel pairings) were detected via one-way ANOVA followed by multiple comparisons between the relative coherence changes using Tukey's Honestly Significant Difference Procedure (Tukey's HSD) to determine if the significant changes in coherence occurred in specific behavioral epoch types or during the post-session optical stimulation period (Fig. 4a, b). $p$ value was corrected using Bonferroni correction to counteract the problem of multiple comparisons.

\section{Spatiotemporal coherence changes between all contact sites (basalo-cortical, cortico-cortical) using Monte Carlo permutation test (Fig. 5)}

For comparison across structures, continuous wavelet coherence (CWC) was calculated between each BF LFP recording site and each cortical recording site (basalo-cortical coherence), and between each V2 site and each OFC contact site (cortico-cortical). Confidence intervals for the event-related cross-coherence were calculated in space and time by Monte Carlo permutation tests or random permutation tests. First, we made a merged distribution of all CWC of LFPs between all combinations of channels from resting "center' behavior epoch (2000 trials) combined with all trials from the event-related coherence. Next, we randomly sampled trials 
Fig. 3 Firing modulation of $\mathrm{BF}, \mathrm{OFC}$ and V2 cells during various behavior epochs (cue presentation, decision making, reward approach). The neurons were selected based on their firing pattern to the specific behavior from a pool of 147 V2 cells, 246 cells in OFC, and 137 cells in BF. a.1 Individual $\mathrm{Z}$-scores of responses of the $\mathrm{BF}(n=6)$, OFC $(n=9)$, and V2 $(n=27)$ neurons during cue presentation. b.1 The individual mean response of the same population of $\mathrm{BF}$ (green upper), OFC (red middle), and V2 (blue lower) neurons with +-STD. c.1 The bars represent the comparison between the normalized firing rate of baseline and during cue presentation. Increased mean firing rate can be observed in V2 (Wilcoxon rank-sum test $p<0.001)$. a.2 Individual Z-scores of responses of the BF $(n=7)$, OFC $(n=17)$, and V2 $(n=4)$ neurons during decision making. b.2 the individual mean response of the same population of BF (green upper), OFC (red middle), and V2 (blue lower) neurons with +-STD. c.2 During the decision making, there was significantly increased neuronal activity in $\mathrm{BF}$ (Wilcoxon rank-sum test $p<0.001$ ), OFC (Wilcoxon rank-sum test $p=0.0012$ ), and V2 (Wilcoxon rank-sum test $p=0.0482)$. a.3 Individual $\mathrm{Z}$-scores of the response of $\mathrm{BF}$ $(n=6)$, OFC ( $n=9)$, and V2 $(n=3)$ neurons during approach reward. b.3 the individual mean response of the same population of BF (green upper), OFC (red middle), and V2 (blue lower) neurons with +-STD. c.3 During approach reward, population activity of OFC (Wilcoxon rank-sum test $p=0.048)$, and V2 (Wilcoxon rank-sum test $p=0.0261)$ significantly increased. Stars above the bars mark the significance $p<0.05$. Cell ID in green bold in (A.2) and (A.3) are cholinergic neurons
A.1

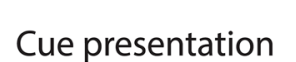

A.2

Decision making

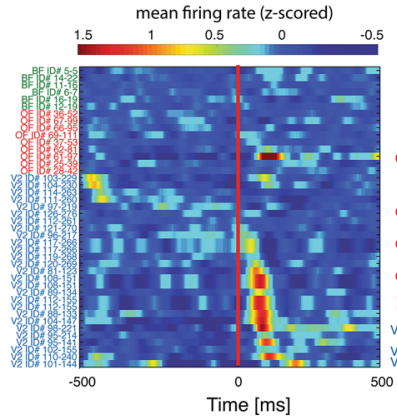

B. 1
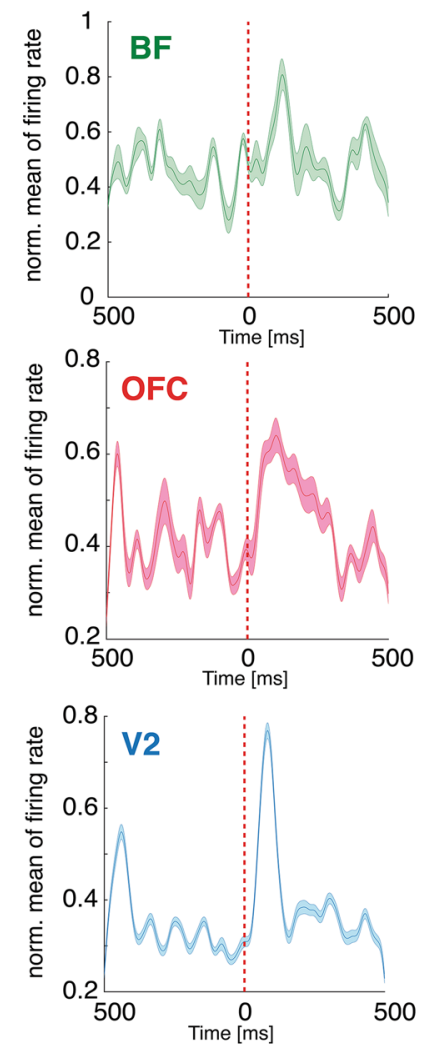

C. 1
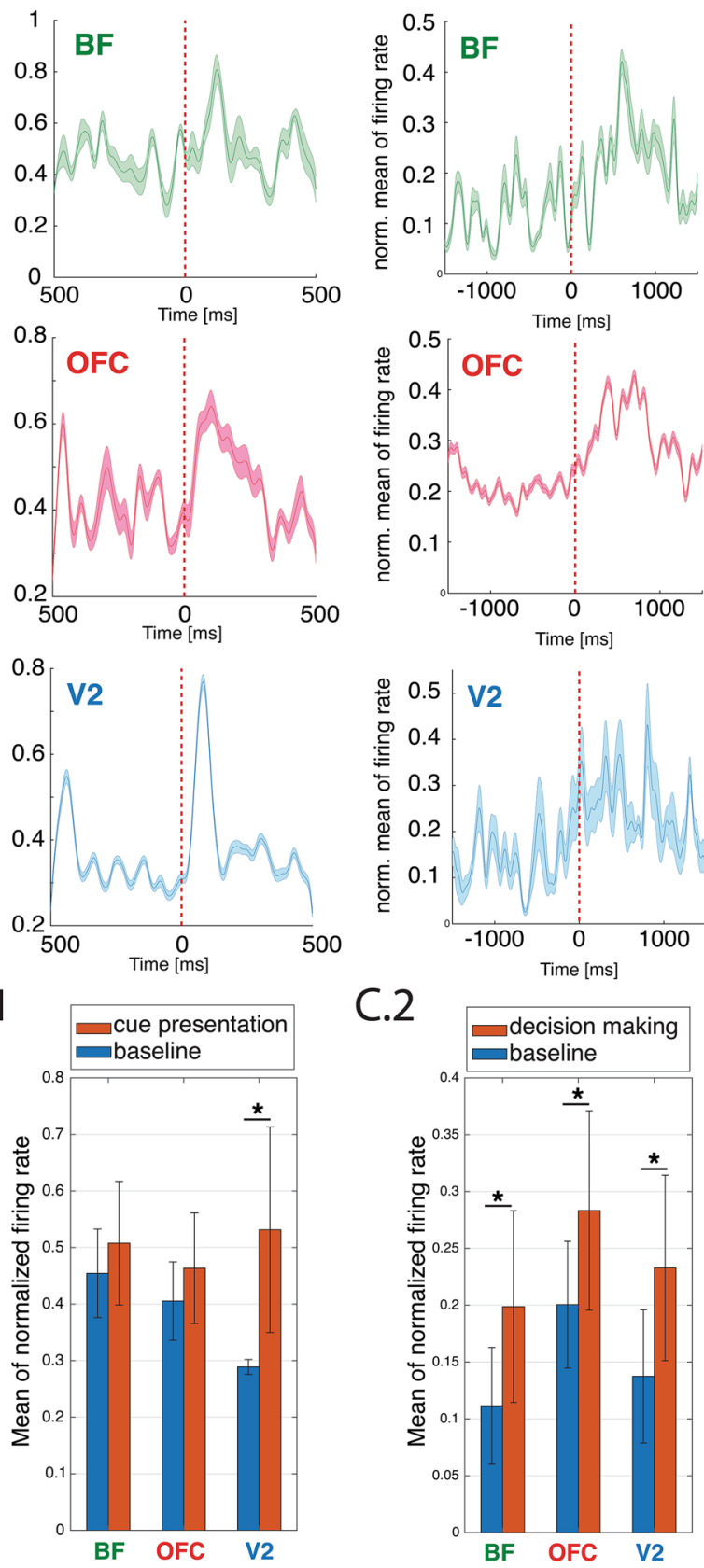

C. 2

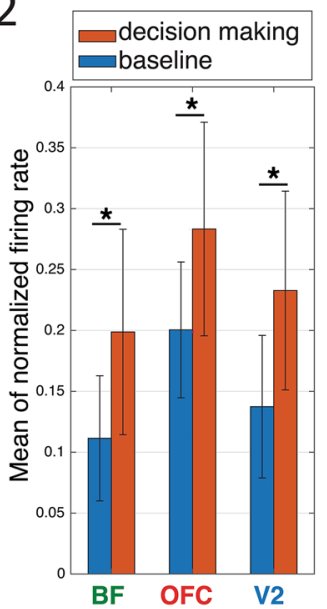

A. 3

Reward approach

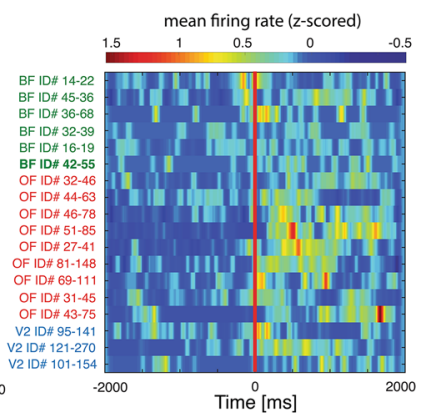

B. 3
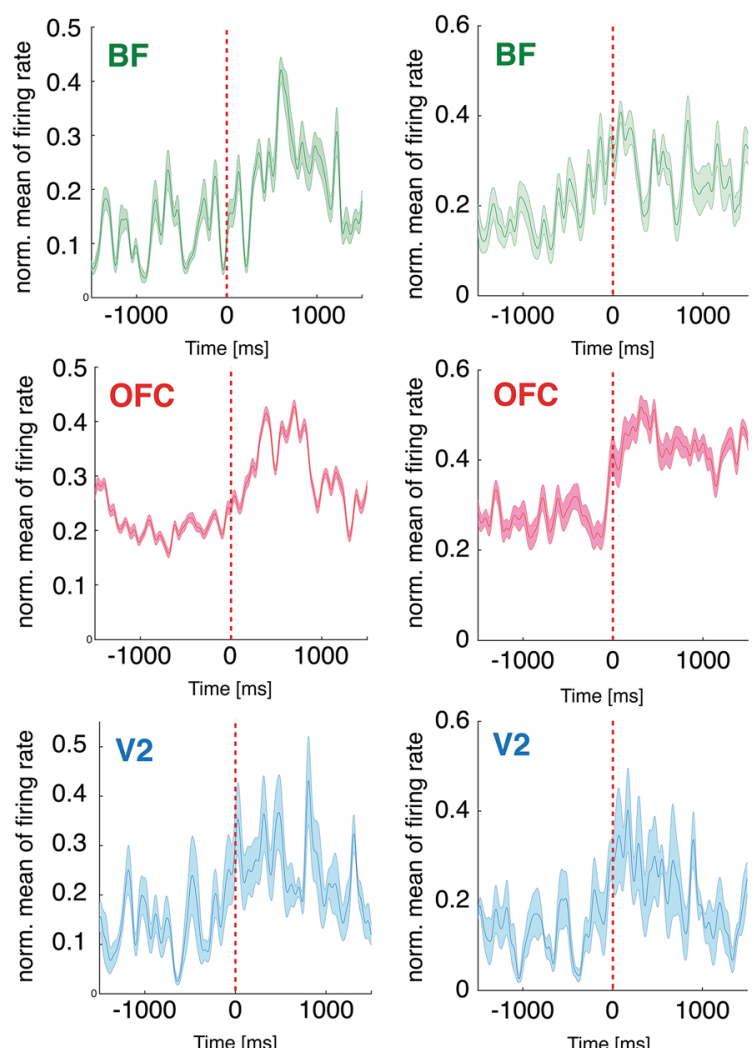

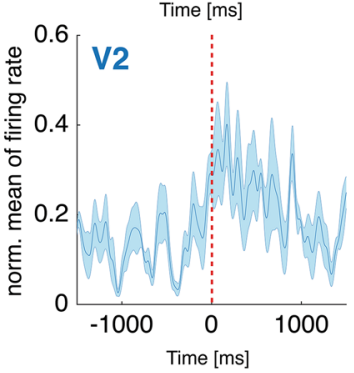

C. 3

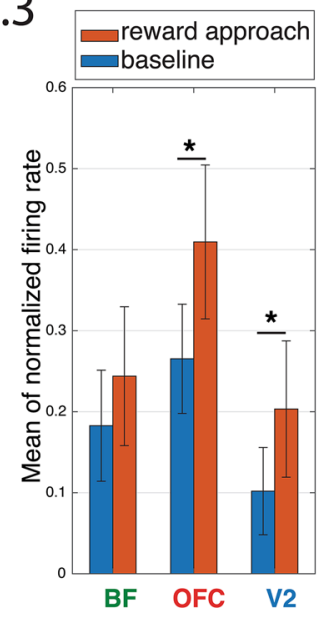


of CWC of LFPs from the combined distribution and then we calculated the mean coherence of both populations, and then calculated the difference score between the two means of coherence This random sampling procedure was repeated 3000 times to create a distribution of statistic values. Finally, we compared the observed coherence change score to the distribution that was generated by the result of the bootstrapped values. If the observed difference was greater than the $95 \%$ of the bootstrapped values, we rejected the null hypothesis that the two behavioral conditions resulted in the same changes in coherence between the given combination of channels $(n)$. the statistical threshold for significant detection of outliers from baseline. A putative connection was considered significant when at least two consecutive bins in the cross-correlograms within +1.5 to +5 ms passed the statistical threshold (Senzai and Buzsáki 2017).

\section{Unit classification based on physiological features (Fig. S4c-i)}

Cholinergic units were classified based on the trough-topeak latency (TP latency) and the first two principal components of the second derivative of their waveform using

Coherence change score $_{n}=\frac{\text { mean }(\text { CWC of BEHAVIOR })-\text { mean }(\text { CWC of RESTING })}{\text { mean }(\text { CWC of BEHAVIOR })+\text { mean }(\text { CWC of RESTING })}$

The coherence change scores were used for constructing a functional spatial map between BF and cortical locations. The analysis was also repeated between the resting and optically stimulated time periods.

\section{Spike-triggered averaged LFP (Fig. 6)}

The oscillatory pattern of cortical areas modulated by cholinergic input was calculated by averaging cortical LFPs, triggered by BF cholinergic spiking. We calculated the empirical cumulative distribution function of the collected power value in low-gamma and high-gamma bands from the continuous wavelet-transformation of the cortical LFPs $\pm 500 \mathrm{~ms}$ around cholinergic spikes. A two-sample Kolmogorov-Smirnov test was applied to compare the empirical cumulative distribution of gamma power before and after cholinergic spikes to reveal significant differences (Fig. 6c, g).

\section{Detection of short timescale interactions of neuron pairs and physiological features (Fig. S4 a, b)}

Cross-correlograms of spike trains of neuron pairs can reveal putative synaptic connections between them (Barthó et al. 2004; Fujisawa et al. 2008; Stark and Abeles 2009). This takes the form of short time-lag (1-6 ms) peaks/troughs with positive or negative deviations from baseline, indicating putative excitatory or inhibitory connections, respectively. Such detection is based on testing the null hypothesis of a homogeneous baseline at a short time-scale (Stark and Abeles 2009). To this end, cross-correlograms binned in 0.5-ms windows were convolved with a $10-\mathrm{ms}$ standard deviation Gaussian window resulting in a predictor of the baseline rate. At each time bin, the 99.99th percentile of the cumulative Poisson distribution (at the predicted rate) was used as the k-means clustering method. To calculate the TP latency, the averaged waveforms were taken from those recording sites where the amplitude shows the maximum deviation. The same averaged waveforms were used to perform PCA using the time period between 0 and $0.8 \mathrm{~ms}$ from the second derivative of the up-sampled averaged waveform. As a result of the waveform PCA, w-PCA1 and w-PCA2 were obtained. Burst index was determined by calculating the average number of spikes in the 3-5 ms bins of the spike autocorrelogram divided by the average number of spikes in the 200-300 ms bins. For k-means clustering, only the TP latency, w-PCA1, and w-PCA2 features were used. Group assignments resulting from k-means were used for supervised learning methods as labeled data. In order to cross-validate the k-means clustering, the TP latency, w-PCA1, w-PCA2 and mean firing rate were all used as features to train the supervised decision tree model (Bootstrap Aggregation "bagging" of Decision Trees model; MATLAB-Learner App., MathWorks, Natick, MA). The cholinergic cells were not part of the training set. With this method, we ensured that our $\mathrm{k}$-means classified data is well-separable. Later, the model was used to predict the k-means group labels for identified cholinergic cells.

\section{Statistical tests used}

In Fig. 4a1-2 b1-2, ANOVA and Tukey's HSD test were used to reveal whether specific behavioral epochs and optical stimulation are linked to changes in BF-cortical coherence versus baseline. For Fig. 5, Monte Carlo permutation tests or random permutation tests for each pairing of recording sites helped us reveal significant anatomical location-dependent coherence differences that depended on the behavior or on optic stimulation of BF. The Kolmogorov-Smirnov (KS) test was used to compare gamma-band power values within the cortex before and after cholinergic spikes recorded from $\mathrm{BF}$ 

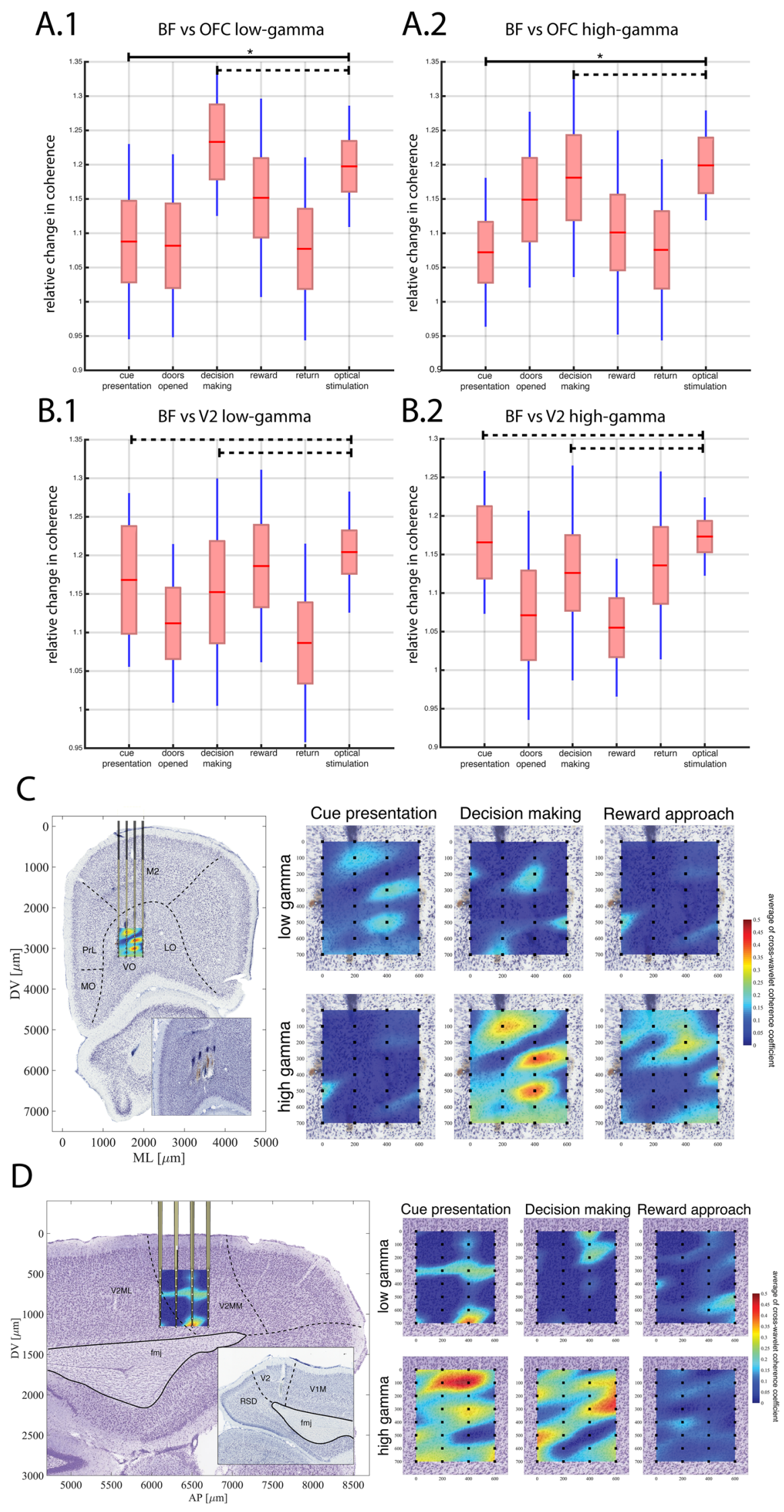
4Fig. 4 Mean of relative changes in coherence at low-low gamma band and in high gamma band between BF-OFC (a.1, a.2), and BF-V2 (b.1, b.2) during different behaviors and optical stimulation. Each bar indicates the median (the central red line), the $95 \%$ confidence intervals (box surrounding the red line) \pm 1 SD. a.1 Effect of behavior epoch and optic stimulation on relative coherence change between OFC vs BF at low-gamma band $\left(F_{(5,119)}=4.96, p=0.0001\right)$. The relative change in coherence during cue presentation significantly differed from the optically stimulated values (Tukey's HSD, $p=0.049$ solid black line above bars). The decision-making condition did not significantly differ from the optically evoked coherence (Tukey's HSD $p=0.96$ dashed black line above bars). a.2 There was a significant effect on the relative coherence change of high-gamma coherence $\left(F_{(5,131)}=4.13, p=0.0016\right)$. Relative change in coherence during cue presentation was significantly different from the optically evoked values at the high-gamma band between OFC and BF (Tukey's HSD $p=0.011$ solid black lines above bars). However, during decision making, the relative change in coherence was not significantly different during the optical stimulation condition (Tukey's HSD, $p=0.99$ dashed black line above bars). b.1 There was a significant effect on the relative change in coherence between BF-V2 at low gamma band $\left(\mathrm{F}_{(5,116)}=3.57, p<0.0049\right)$. However, coherence changes during cue presentation (Tukey's HSD, $p=0.99$ ) and decision making (Tukey's HSD, $p=0.85$ ) did not differ from the relative change in coherence during optical stimulation. b.2 There was a significant effect on the relative coherence change between BF-V2 at high gamma $\left(F_{(5,116)}=3.47, p<0.0058\right.$ ), but the cue presentation (Tukey's HSD, $p=0.99$ ) and decision making (Tukey's HSD, $p=0.96$ ) did not differ from the relative change in coherence during optic stimulation. c Coronal section showing orbital tracks of contact sites (black dots 4X8) of linear silicon electrode arrays, overlaid by spatially localized mean of cross-wavelet coherence at high gamma band between BF and OFC contact sites. Inset shows a coronal section with electrode tracks. The black dots on each surface plot represent the contact sites from $\mathrm{OFC}$ silicon electrode arrays. $\mathrm{X}-\mathrm{Y}$ axes show the relative distance between contacts in $\mu \mathrm{m}$. d Sagittal section showing V2 contact sites (black dots) of linear silicon electrode arrays, overlaid by spatially localized mean of cross-wavelet coherence at low gamma band between $\mathrm{BF}$ and V2. Inset shows a coronal section with the electrode tracks. The black dots on each surface plot represent the contact sites from V2 silicon electrode arrays. $\mathrm{X}-\mathrm{Y}$ axes show the relative distance between contacts in $\mu \mathrm{m}$

(Fig. 6). The main question was whether or not the distribution of gamma power at certain locations in the cortex in the period before $500 \mathrm{~ms}$ and after $500 \mathrm{~ms}$ of the cholinergic spike is the same or not? Did it change or not? If KS test shows significant differences, the power of gamma values comes from a different distribution (Fig. 6c, g). Thus, the firing of a cholinergic cell modulated the gamma power at specific locations in the cortex, but not globally.

\section{Results}

\section{Anatomy of cholinergic projections to OFC and V2 (Fig. 2)}

To demonstrate the cholinergic projection from the basal forebrain to OFC and V2, we used retrograde viral tracing. Although cholinergic neurons projecting to V2 are located throughout the whole extent of the diagonal band of Broca/ substantia innominata (Huppé-Gourgues et al. 2018), OFCprojecting cholinergic cells tend to concentrate more caudal in the BF, including the internal capsule and adjacent globus pallidus region (Gielow and Zaborszky 2017). In one case, using monosynaptic virus-tracing, cholinergic neurons that collateralized to both $\mathrm{OFC}$ and V2 were found from -0.4 to $-2.8 \mathrm{~mm}$ to the bregma (Paxinos and Watson $2007)$ in the internal capsule and adjacent globus pallidus $(n=13)$. The distribution of the various types of labeled neurons from this case are summarized in Supplementary Table S1. A comparison between cholinergic-specific innervation of OFC and V2 and the location of labeled cells in the BF from various experimental cases from the Allen Brain Institute (Allen 2011) also suggests that these two cortical areas receive joint projections from a limited $\mathrm{BF}$ area (see Fig. 2).

\section{Physiological properties of cholinergic and noncholinergic cells from BF, OFC and V2 (Figs. S2-S4)}

We categorized recorded cells using a combination of techniques: a quantitative classifier based on the waveform and spiking cross-correlations to identify putative excitatory and inhibitory cells in the BF and in OFC and V2, and optogenetic tagging for identification of cholinergic cells in the BF (Fig. S4). First, the short-latency temporal interaction between all neuron pairs, using their spiking cross-correlation, identified excitatory and inhibitory units (Barthó et al. 2004) based on short ( $<5 \mathrm{~ms}$ ) latency offsets between two recorded neurons. The putative inhibitory and excitatory cells were grouped by trough-to-peak latency (TP-latency) using filtered waveforms (Fig. S4a. inset) and burst index (Fig. S4a). Combining these features (putative excitation and inhibition) with TP-latency resulted in three major groups of cells: excitatory cells (BF $n=19 / 137$; OFC $n=159 / 246$; V2 $n=109 / 147)$, narrow-waveform inhibitory cells (BF $n=89 / 137$; OFC $n=28 / 246$; V2 $n=7 / 147$ ), and widewaveform inhibitory cells (BF $n=0 / 137$; OFC $n=18 / 246$; $\mathrm{V} 2 n=11 / 147)$. The mean firing rates of excitatory neurons in $\mathrm{BF}($ median $=5.8304 \mathrm{~Hz})$, in OFC $($ median $=3.935 \mathrm{~Hz})$ and in V2 (median $=5.275 \mathrm{~Hz}$ ) were not significantly different $(p>0.05)$. The average firing rate of cholinergic neurons was $2.16 \mathrm{~Hz}$ and their average TP-latency was $0.6062 \mathrm{~ms}$. Overall, the waveforms of cholinergic cells fell in the widewaveform excitatory group, non-cholinergic neurons showed great heterogeneity and were classified in six different groups using K-means clustering and a supervised 'bootstrap aggregating' machine learning meta-algorithm (Fig. S4). Spiking activity of individual neurons from BF, OFC, and V2 are shown in Figs. S2, and Fig. S3. 
A

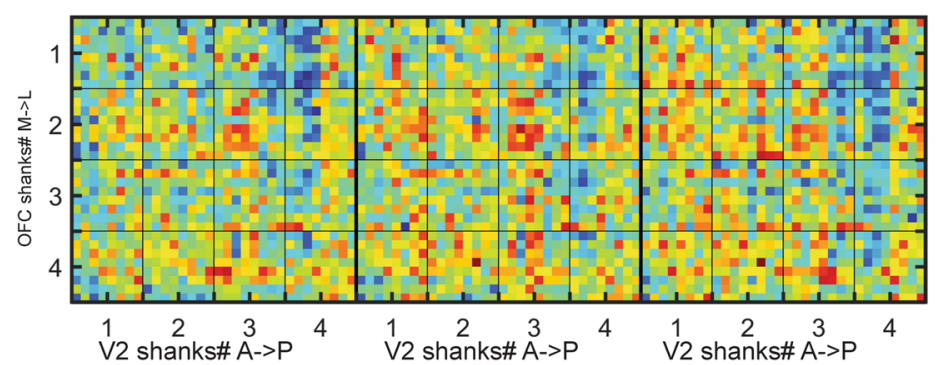

average cortico-cortical coherence change at high- $\gamma$
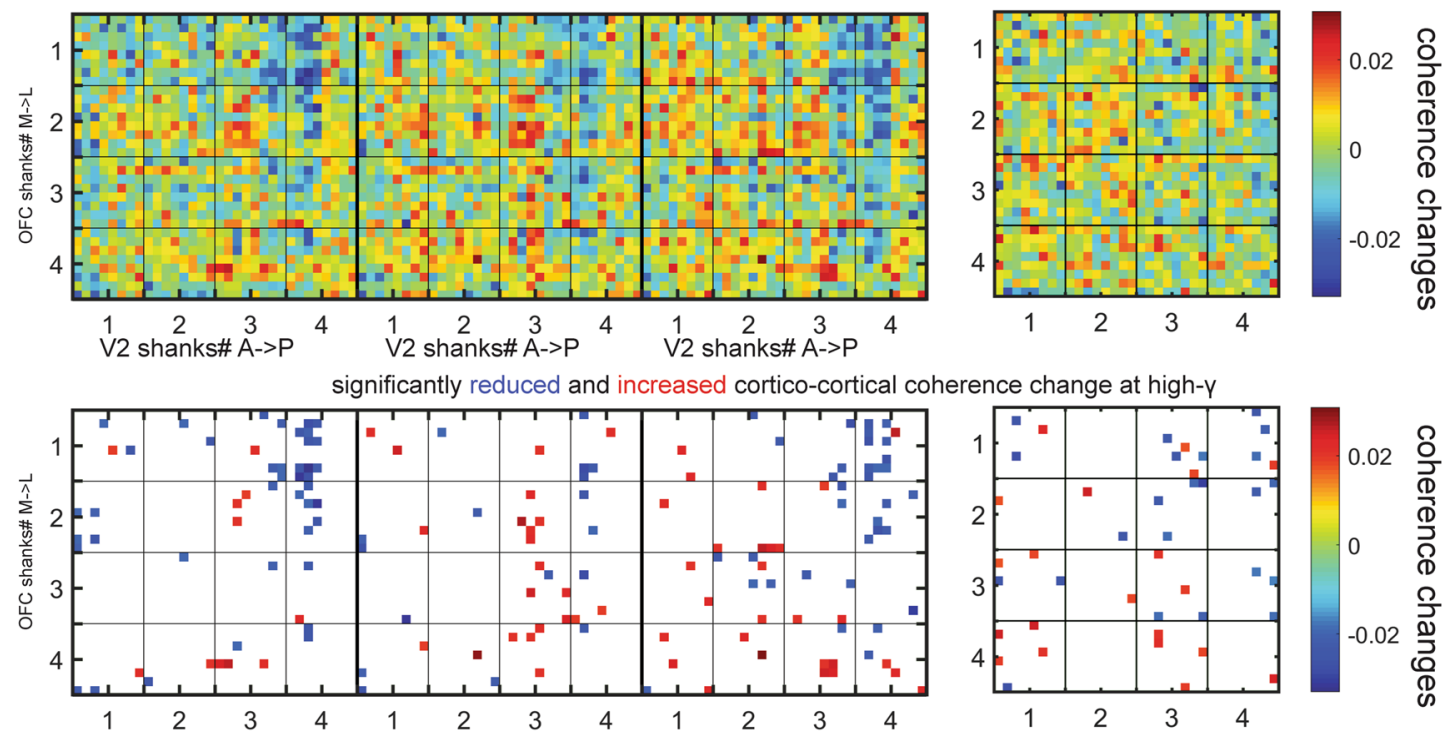

B
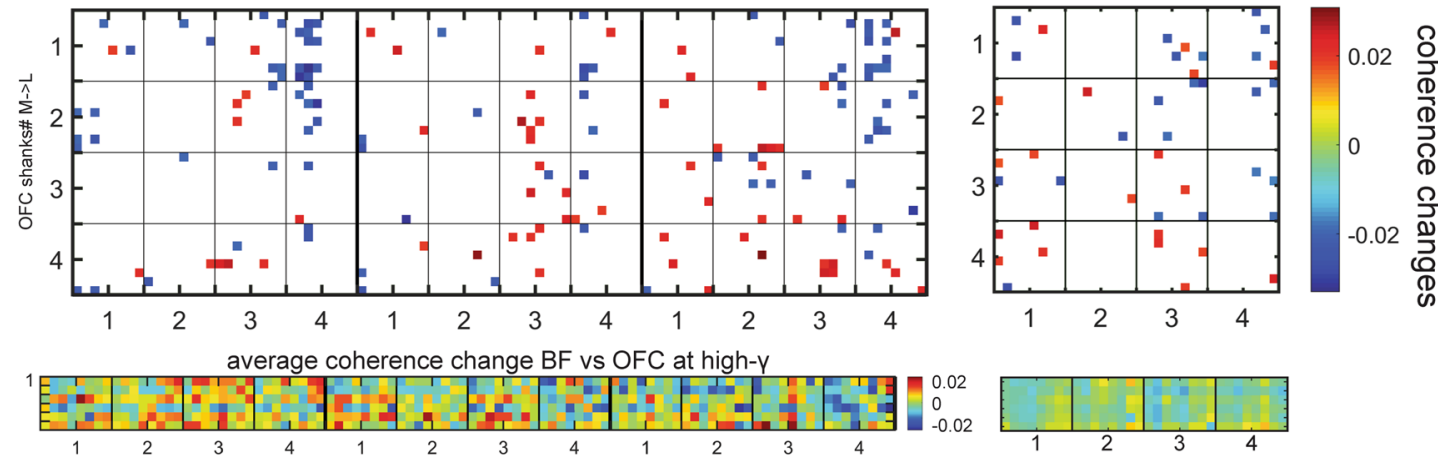

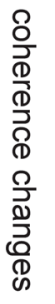
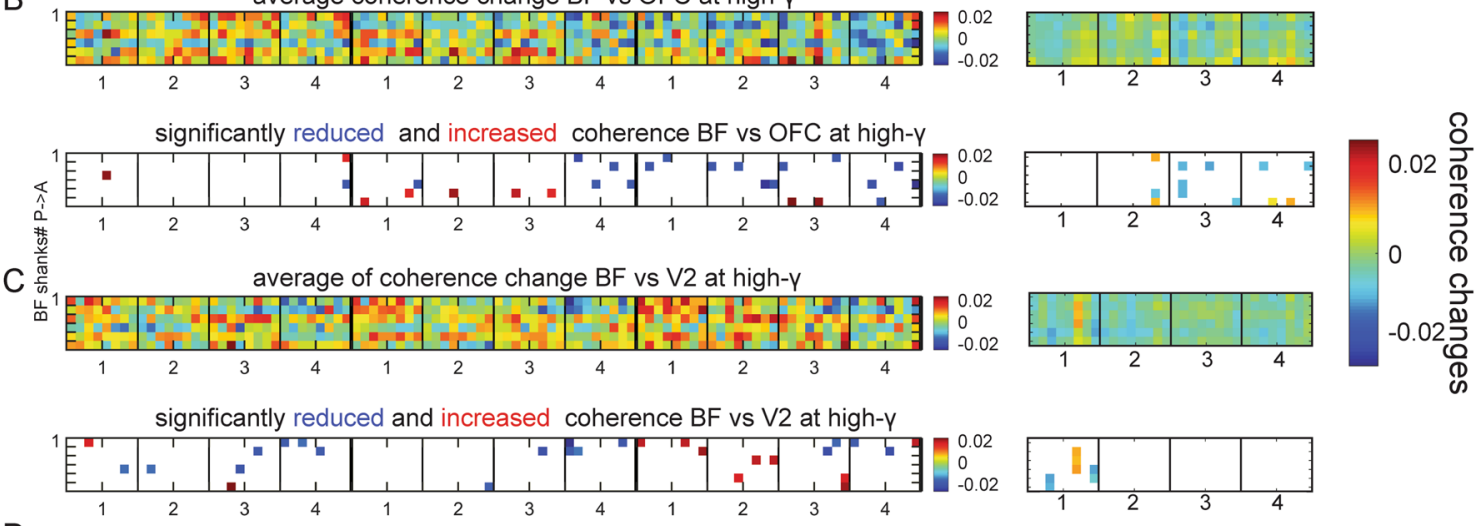

D

significantly changed basalo-cortico-cortical coherence at high- $\mathrm{Y}$

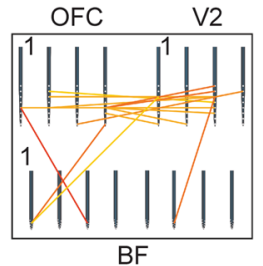

BF

Visual stimuli

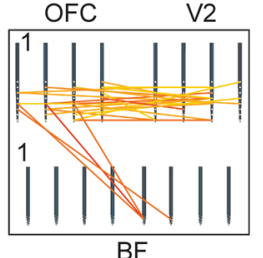

Decision making

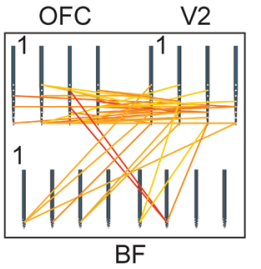

Reward approach

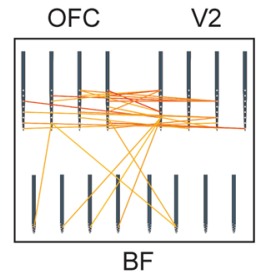

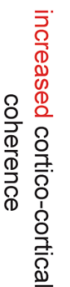

Blue light ( $473 \mathrm{~nm})$ stimuli of $B F$
Fig. 5 Maps of basalo-cortical and cortico-cortical high gamma band coherence changes during behavior. The three columns of each subplot divided by thick lines represent the behavioral epochs left to right: cue presentation, decision making, reward approach. Each $32 \times 32$ matrix represents a coherence map between electrode contacts on shanks (shanks\#: $x$-axis V2, y-axis OFC). a The upper plot is the mean of cortico-cortical coherence change; the lower plot is the significant coherence change map. b upper plot: BF vs OFC coherence change; lower plot significantly changed coherence map, c upper plot: BF vs V2 coherence map; lower plot significantly changes coherence map. d Significantly increased spatiotemporal basalo-cortical and cotico-cortical-coherence maps using schematic arrangement of electrode locations representing contact pairs between OFCV2-BF. In each box, lower 8 lines BF, upper left lines 4 OFC and upper right $4 \mathrm{~V} 2$ shanks. The fourth column represents data during blue light stimulation of BF using the same illustration method as during behavior in the left three columns. Color bars to the right indicate the extent of changes 

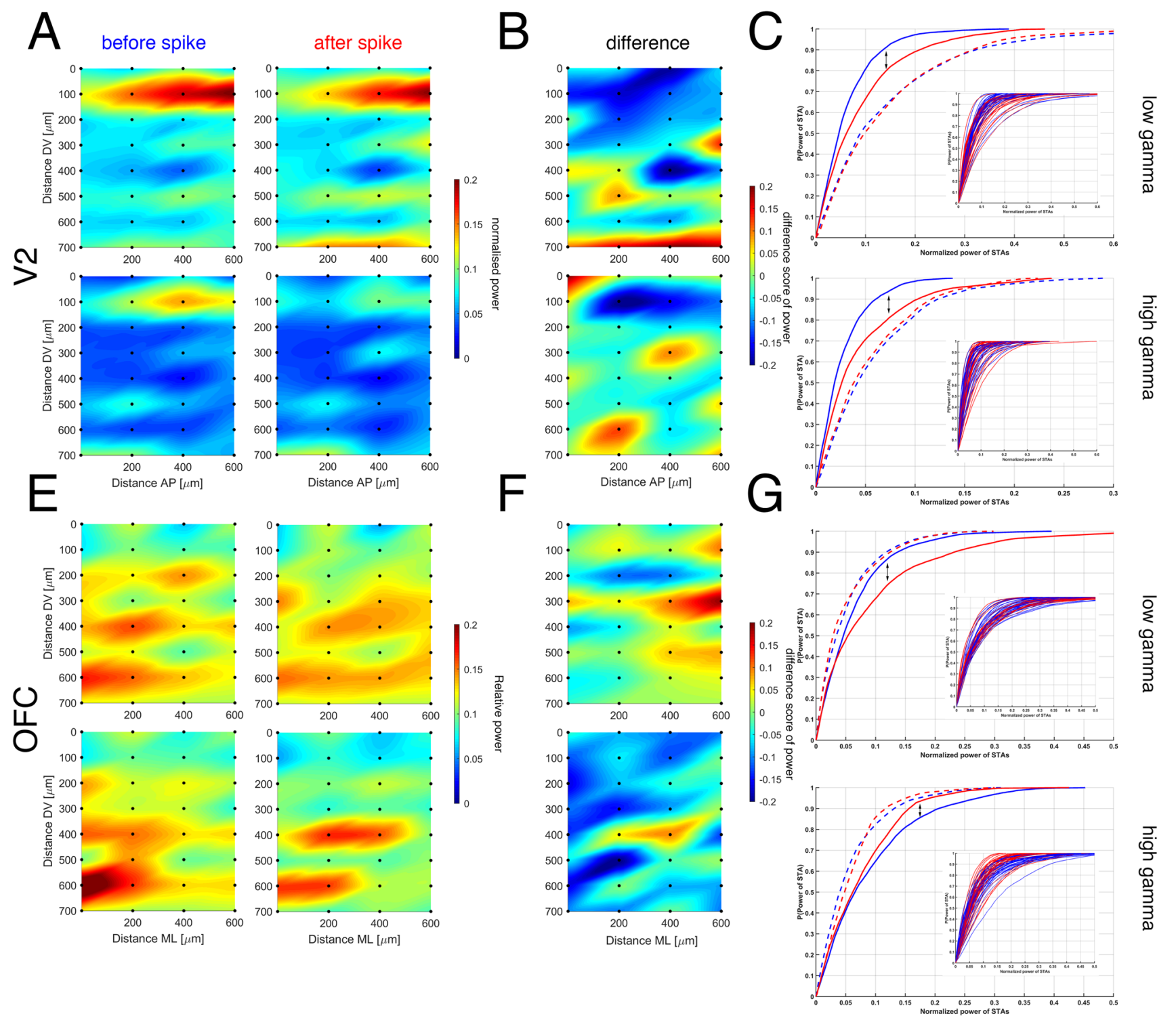

Fig. 6 Plots of spike-triggered averages (STA) of the local field potentials from V2 (a), and from OFC (e) at specific cortical electrode sites (black dots) triggered by cholinergic cells (BF ID 42-55). The first row of a shows the power of STA at low gamma band from $\mathrm{V} 2$ and the first row of e corresponds with the low gamma power of STA from OFC, in contrast, the second row of a and e shows high gamma activity for given structures. "Before" above the first column with (blue) and "after" above the second column (red) represent the spatial gamma power activity before the spike and after the spike. The extracted LFPs time windows were 500-500 ms. Panels b and f show the difference score of STAs at low, and high gamma. c V2 and $\mathbf{g}$ OFC show the exponential cumulative distribution of low and

\section{Cell responses during cue presentation, decision making and reward approach (Fig. 3)}

To characterize the firing modulation of neurons in BF, OFC, and V2, we examined neuronal population responses during different behavioral epochs. During the task, animals high gamma power triggered by cholinergic spikes before the spike (blue) and after (red) lines. The continuous lines show the significant $(p<0.01)$ difference between cumulative distribution functions of the exponential distributions of the power before and after the cholinergic spike at a specific location. In contrast, the dashed lines show a non-significant differences between the distributions. The subplots show the distributions for each of the channels before (blue) and after the cholinergic spikes (red) lines at low gamma (first row) and high gamma frequency (second row) bands from V2 (c) and OFC (g). Dorso-ventral (DV) and antero-posterior (AP) location of contact sites are indicated on the $\mathrm{Y}$ and $\mathrm{X}$

were required to recognize and allocate attention to detect the frequency of visual stimulus presented on the doors of the center of the radial arm maze (Fig. 1b). After the doors opened for both directions, the animal decided to use one of the arms to approach the reward. 


\section{Visual cortex}

During the cue presentation, the firing rate in some V2 cells changed relative to the condition when the animal remained in the center without cues (27/147; Wilcoxon rank-sum test; $p<0.05$ criterion; Supplementary Fig. S2c, representative PSTHs in Supplementary Fig. S3d, e). More specifically, two cells showed ON-OFF responses (Fig. 3a1 ID\#103-229; 104-230), two cells increased their firing rate during the offset of the stimuli (Fig. 3a.1 ID\#114-263; 111-260), and the rest of the 23 neurons in V2 showed increased firing rate to stimulus onset (Fig. 3a.1). Before stimulus onset (baseline) the normalized (feature scaling) average firing rate of the V2 population was $0.2889,+-\mathrm{STD}=0.0132$, in contrast, during the visual cue (Fig. 3c.1), the normalized average firing rate was 0.5314 ; +-STD 0.187 . The population activity significantly increased during cue presentation (Wilcoxon rank-sum test $p<0.001)$. The population activity also significantly increased during decision making ( $n=4$ Fig. $3 c .2$, baseline normalized mean of firing rate: $0.1450+-$ STD: 0.0728 , during decision making the normalized mean of firing rate: $0.2492+-$ STD: 0.0926; Wilcoxon rank-sum test $p=0.0482)$ and reward approach ( $n=3$ Fig. $3 c .3$ baseline normalized average population firing rate of neurons: 0.1384 , during reward approach: 0.2229 , Wilcoxon rank-sum test $p=0.0261)$.

\section{Orbitofrontal cortex}

In the OFC, we encountered cells that were modulated during cue presentation (Fig. 3a.1) but not significantly at the population level (9/246 norm. average of firing rate before cue presentation (baseline): $0.4053+-\mathrm{STD}$ : 0.0693, 0.4633 + -STD: 0.0979 during visual cue); however, cells significantly increased firing during decision making (Fig. 3c.2: 17 cells out of 246; baseline average normalized population firing rate before decision making: $0.2122+-$ STD: 0.0384; during decision making: $0.2993+-S T D: 0.07$; Wilcoxon rank-sum test $p=0.0012$ ), and also increased significantly during reward approach (9/246 Fig. 3c.3 baseline normalized average firing rate before reward approach: $0.2798+-$ STD: 0.0527 and during reward approach: 0.4403+-STD: 0.0523, Wilcoxon ranksum test $p=0.048$ ).

\section{Basal forebrain}

$\mathrm{BF}$ cells showed heterogeneous responses. In the spike train of the BF (Supplementary Fig. S1f), two types of activity can be observed: cells with high firing rates and those with very low firing rates $(<1 \mathrm{~Hz})$. Those cells showing low firing rates were identified as cholinergic based on optogenetic tagging. One cholinergic neuron significantly increased its firing during the reward approach (1/137; BF ID\#42-55 in Fig. 3a.3 and in Supplementary Fig. S2 \#55). Another cholinergic neuron showed increased firing during decision making (BF ID\#14-22 Fig. 3a.2, Supplementary Fig. $\mathrm{S} 3 \mathrm{a} \# 14-22$ ). We found a few non-cholinergic neurons that were modulated by cue presentation, but their normalized averaged firing rate did not change significantly (6/137, Fig. 3c.1). During decision making (7/137; Fig. 3a.2, b.2) their normalized average firing rate together with cholinergic neurons \#14-22 was $0.1170+-$ STD: 0.0638 before decision making (baseline), which significantly increased to $0.2101+-$ STD:0.0914 during decision making (Fig. 3c.2 Wilcoxon rank-sum test $p<0.001)$. Together with other BF neurons (6/137), the averaged normalized firing rates were $0.1938+-$ STD: 0.0729 before the reward approach and $0.2567+-$ STD: 0.0884 during the reward approach: (Fig. 3B.3, C.3).

\section{BF-OFC and BF-V2 relative coherence changes are similar between decision making and during optical stimulation (Fig. 4)}

Oscillations in neuronal circuits can spatiotemporally coordinate the transmission of information. To address the temporal dynamics of oscillatory interactions between $\mathrm{BF}$ and cortex, basalo-cortical coherence changes were calculated using LFPs from the BF, OFC and V2 (see methods). To decrease the complexity of the investigations, we focused on the low and high gamma bands for further analysis. Supplementary Fig. S1a, b, and c show representative continuous wavelet transformation (CWT) of LFPs from the three regions during a single trial between 1 and $110 \mathrm{~Hz}$. To address the variability between animals and between trials within a rat, the coherence values of behavioral epochs and during optic stimulations were normalized as a proportion of baseline. Baseline coherence values were measured when the animals were resting, positioned in the center of the maze, with closed doors for each trials. We concatenate the values of the proportion of coherence of each trials, from the forty-five sessions (averaged trial number: 15/session SD- +2.3) of the three animals.

\section{BF-OFC relative coherence changes}

The relative $\mathrm{BF}-\mathrm{OFC}$ coherence changes in both the high and low gamma ranges were elevated during the decision-making behavioral epoch of the task (Fig. 4a.1-a.2). To compare changes in coherence across each behavioral epoch and optical stimulation, a one-way ANOVA was conducted. We found that there were significant coherence changes within the low gamma band for the six conditions (Fig. 4a.1; $\left.F_{(5,119)}=4.96, p=0.0001\right)$. Next, we performed multiple comparisons (Supplementary Table $2 \mathrm{a}-\mathrm{b}$ ) between 
the relative coherence changes using Tukey's Honestly Significant Difference Procedure to determine if the significant changes in coherence occurred between particular behavioral epochs (cue presentation, decision making, etc.). Post hoc comparisons indicated that the relative coherence change of the BF-OFC low-gamma band during optical stimulation significantly differed from the coherence change during cue presentation (optical stimulation $\bar{x}=1.197 \pm 0.027$; cue presentation $\bar{x}=1.087 \pm 0.027, p=0.049)$ but not during decision making ( $\bar{x}=1.2332 \pm 0.033, p=0.96$; Fig. 4A.1; solid and hyphenated lines above the individual bars). There was also a significant effect on the relative coherence change of high-gamma coherence between BF and OFC $\left(F_{(5,131)}=4.13, p=0.0016\right.$; Fig. 4a.2). Post-hoc comparisons revealed that the relative coherence change during the optical stimulation differed significantly from the coherence change during the cue presentation epoch (cue presentation $\bar{x}=1.072 \pm 0.026$; optical stimulation $\bar{x}=1.189 \pm 0.024$; $p=0.011$ ). However, similar to the low gamma band comparisons, the relative coherence change during decision making did not differ significantly from the optically evoked coherence change at the high gamma band (decision making $\bar{x}=1.181 \pm 0.027 ; p=0.99$; Fig. 4a.2). Taken together, these results suggest that optically evoked changes in relative coherence between $\mathrm{BF}$ and $\mathrm{OFC}$ were similar to the relative change in coherence during decision making, and they differed from the change in relative coherence during cue presentation.

\section{BF-V2 relative coherence changes}

There was a significant effect on the relative coherence change between BF-V2 at low gamma band for the five behavioral epochs and optical stimulation (Fig. 4b.1; $\left.F_{(5,116)}=3.57, p<0.0049\right)$. However, the post hoc analysis (Supplementary Table $3 \mathrm{a}-\mathrm{b}$ ) indicated that the relative change of the optically evoked coherence $(\bar{x}=1.204$ $\pm 0.0276)$ did not differ from the relative change in coherence during cue presentation (optical stimulation $\bar{x}=1.204 \pm 0.0276$; cue presentation $\bar{x}=1.168 \pm 0.028$; $p=0.99)$ or during decision making $(\bar{x}=1.152 \pm 0.021$; $p=0.85$ ). Similarly, at the high gamma band, there was a significant difference between the relative change in coherence (Fig. 4b.2; $\left.F_{(5,116)}=3.47, p<0.0058\right)$. However, the visual cue $(\bar{x}=1.165 \pm 0.029 ; p=0.99)$ and decision making $(\bar{x}=1.126 \pm 0.02 ; p=0.96)$ behavioral epochs did not significantly differ from the optically evoked change in coherence $(\bar{x}=1.153 \pm 0.026)$.

The above statistical analysis revealed similarities between specific behavioral epochs and optic stimulation. Beyond the temporal dynamic changes in coherence during the behavioral epochs, we aimed to reveal whether the coherence displays homogeneous or spatially focal maxima between the BF and the cortex. Panels in Fig. 4c-d demonstrate behavior-dependent spatially localized coherence events; we calculated averaged wavelet coherences between a single BF contact site (mean of LFPs of contact sites of 5th shank) vs the OFC at each contact site (individual LFPs of all cortical contact sites). Figure $4 \mathrm{c}$ demonstrates the spatiotemporal "co-oscillation channels" around localized contact sites in the OFC within each behavior epoch, including decision making and reward approach at low and high-gamma bands. The coherence at the high-gamma band seems to be more robust spatially than during cue presentation or reward approach.

The average BF-V2 coherence has spatially distinct local maxima around specific array contact sites (Fig. 4d). Basalocortical coherence increased in the low-gamma band during cue presentation, and a horizontal coherence "layer" can be observed around $300 \mu \mathrm{m}$ depth. This horizontal-shaped coherence disappeared during the decision-making and reward approach epochs in the low gamma (Fig. 4d, upper row). In the high gamma during cue presentation and decision making, several increased coherence patches can be observed. These results drove us to extend the analysis to investigate spatio-temporal coherence between $\mathrm{BF}$ and $\mathrm{V} 2$, $\mathrm{OFC}$ at all contact sites, as described in the next paragraph.

\section{Cortico-cortical coherence during behavior and cholinergic stimulation (Fig. 5)}

We further analyzed detailed spatial coherence changes between all contact sites in the cortex and each shank in the BF using Monte Carlo permutation tests (Fig. 5). As the previous results revealed, the basalo-cortical coherence was spatiotemporally modulated during decision making simultaneously between BF/OFC and BF/V2 at high gamma band (Fig. 5, panels c and d); therefore, we tested corticocortical coherence changes during behavioral epochs. Each of the coherence change values were extracted between all combinations of contact sites, creating a coherence matrix consisting of 1024 voxels. These voxels represent LFP/LFP coherence changes in the three different behavioral epochs (visual stimulation, decision making and approach reward: Fig. 5a, first row, left matrix) and optic stimulation of BF (Fig. 5a, first row, right matrix). Each combination of contact sites indicates coherence change score; e.g., change between center versus cue presentation, decision making, or approach reward approach. Scores range from -1 (decrease of coherence during behavioral epochs) to +1 (increase of coherence during behavioral epoch). In Fig. 5 panel 'a' depicts the average cortico-cortical coherence change matrix during cue presentation, decision making and reward approach epochs between OFC and V2. To distinguish behavioral or optic stimulation-related coherence score changes, we calculated the critical values for each combination of coherence 
changes to determine the significance level (1\% significance level) using a bootstrapping method (see methods). Figure 5 panel 'a', second row displays only significantly $($ alpha $=0.01)$ reduced (blue) or increased (red) locations of cortico-cortical coherence change at high gamma. Panels in Fig. 5b display average (first row) and significant coherence change (second row) pairs between BF and OFC. Finally, panels in Fig. 5c display average (first row) and significant coherence change (second row) pairs between BF and V2. The bottom row in Fig. 5 (panel d) shows schematically the significant spatio-temporal relations of functional connectivity between $\mathrm{BF} / \mathrm{OFC}$ and $\mathrm{BF} / \mathrm{V} 2$ in the distinct behavioral epochs at high-gamma band with illustration of individual shanks.

It is apparent that the number of significant coherence combinations is different in the specific behaviour epochs. The inhomogeneous spatial distribution of the coherence between the contact sites from the two cortical regions or between BF and cortical sites seems to depend on the behavioral epochs and the anatomical location of probes.

To compare the fine spatial distribution of basalo-cortical and cortico-cortical coherence changes during cholinergic stimulation, the right column of Fig. 5 uses the same type of illustration. The coherence differences suggest that the cholinergic system can modulate cortico-cortical gammarelated coherence patterns, enabling information processing between these cortical regions.

\section{Cholinergic spike-triggered averages of spatial cortical LFP (Fig. 6)}

Spatial spike-triggered averages (STA) of cortical LFPs induced by cholinergic firing demonstrate their effect on cortical gamma power (Fig. 6). We calculated the power of low gamma and high gamma at every contact site in V2 (Fig. 6a) and in OFC (Fig. 6e) before and after the cholinergic spikes within a $[-500,500 \mathrm{~ms}]$ time window. The difference scores showed power changes around spike events, which demonstrated differences of the power in low and high gamma bands in V2 (Fig. 6b), and OFC (Fig. 6f). In V2, the power of low gamma increased significantly around $300 \mu \mathrm{m}$, $500 \mu \mathrm{m}$ and $700 \mu \mathrm{m}$ relative depth within the cortex (Fig. 6b). Similarly, Fig. 6f for OFC shows increased spike-triggered averaged (STA) LFP patches at low and high gamma band.

The empirical cumulative distribution function (ECDF) represents the accumulated power values within a $1000 \mathrm{~ms}$ time window centred on each spike (Fig. 6c for V2 and Fig. $6 \mathrm{~g}$ for OFC). A two-sample Kolmogorov-Smirnov test showed significant differences $(p<0.05)$ between the ECDF of before events, and that of after events across all cortical contact sites (Fig. 6c, g and Table 1). Table 1 summarizes the percentage of significant gamma changes at specific contact sites for all cholinergic cells, where the ECDF showed significant differences. For example, in the case of cholinergic cell ID16-39, one site showed significant differences in V2 (low gamma; Kolmogorov-Smirnov test; $p=0.016$, ks2stat $=0.045$ ) and the same cell displayed significant differences at another site in both low and high gamma, respectively in OFC ( $p=0.041 ; \mathrm{ks} 2 \mathrm{tat}=0.041$ and $p=0.016$, ks2stat $=0.046$ ). Another cholinergic-induced STA analysis (cell ID14-22) shows significant differences at two sites in the OFC at low gamma $\left(p=6.118^{*} 10^{-6}\right.$; ks2stat $=0.104$ and $p=0.003$, ks2stat $=0.075)$ and at high gamma: $p=4.83 * 10^{-15}, \mathrm{ks} 2 \mathrm{stat}=0.169$ and $p=0.015$, ks2stat $=0.064)$.

\section{Discussion}

In this study, we investigated the functional circuitry between specific locations in the BF, orbitofrontal (OFC), and visual cortex (V2) with high-density electrode arrays during a visual discrimination task, performed by transgenic ChAT-Cre rats. It is known from anatomical studies that V2 and OFC are reciprocally connected (Reep et al. 1996), and we have identified in a single case that a few cholinergic neurons in the internal capsule/globus pallidus/substantia innominata region collateralized to innervate both $\mathrm{V} 2$ and the orbitofrontal cortex.
Table 1 Percentage of significant gamma changes at specific contact sites

\begin{tabular}{lllllll}
\hline Cell ID & V2 & & OFC & & $\begin{array}{l}\text { Shank\# } \\
\text { (loca- } \\
\text { tion) }\end{array}$ \\
\cline { 2 - 3 } & Low gamma (\%) & High gamma (\%) & Low gamma (\%) & High gamma (\%) \\
\hline $14-22$ & 0 & 0 & 6.25 & 6.25 & 5 \\
$16-39$ & 3.125 & 0 & 3.125 & 3.125 & 5 \\
$32-49$ & 0 & 0 & 0 & 0 & 7 \\
$42-55$ & 3.125 & 0 & 9.375 & 0 & 7 \\
$43-56$ & 12.5 & 0 & 0 & 0 & 7 \\
$45-61$ & 6.25 & 3.125 & 0 & 0 & 3 \\
\hline
\end{tabular}


In the behavioral task, both in low and high gamma, coherence changed according to the behavioral demands between BF/OFC and BF/V2, respectively (Fig. 4). Moreover, we found distinct differences in the fine spatial distributions of coherence values between basalo-cortical and cortico-cortical sites during specific behavioral epochs (Fig. 5). For example, Fig. 5d reveals a hotspot of coherence between a specific BF and V2 sites (BF 6th shank vs V2 shank 3th) or between a specific BF and OFC sites (BF 6th shank-OFC 3th shank) from the same location in a behavior-dependent manner. It only exists during the specific behavior (during visual stimuli and reward approach). But the BF-V2 coherence, for example, during reward shows more global changes in terms of localization in contrast to visual cue. The spatial coherence pattern maintains across trials, even across sessions in the same behavior epoch and anatomical localization. The spatial map of coherence, however, is not static, but dynamically changes between behavioral epochs. Moreover, laser stimulation of BF cholinergic cells generates spatial coherence changes among specific cortico-cortical sites (Fig. 5 right column), and cholinergic spike triggered averages of cortical LFPs showed significant differences before and after cholinergic spikes at specific cortical contact sites (Fig. 6, Table 1), suggesting that the cholinergic system contributes to selective modulation of cortico-cortical circuits. Across behaviors and task demands, coherence between disparate but connected brain regions dynamically changes under the control of specific BF sites. Mapping the cortical coherence changes together with the location of electrode arrays in the BF (Fig. 5d) suggests that neurons in specific sub-regions in the BF are dynamically recruited during the various behavioral epochs to coordinate specific cognitive processes.

\section{BF oscillatory dynamics and their correlation with discrete cortical oscillations}

Recent large-scale recording of the BF shows that neurons fire with peaks in different frequency ranges that strongly correlate with specific behavioral epochs, including target detection, decision making, and outcome evaluation (Tingley et al. 2014, 2015, 2018). Although these authors seldom used simultaneous recording in the BF and related cortical areas, they suggested that these 'nested' oscillations in the BF ('multiplexing') might maximize the transfer of segregated information to remote cortical regions in a sequentially organized fashion. In our task, low and high gamma (40-100 Hz) dominated the three investigated brain regions in all behavioral epochs, except for a strong theta power in V2 (Supplementary Fig. S1).

There is a vast literature to suggest that neuronal interactions are modulated through synchronization (Womelsdorf et al. 2007). Gamma-band synchronization involves rhythmic inhibition of local cortical networks (Cardin et al. 2009;
Kim et al. 2016); the periods between inhibition provide temporal windows for neuronal communication. If a stimulus is selected by attention, communicating cortical areas show stronger and higher frequency gamma-band synchronization (Lakatos et al. 2008; Gregoriou et al. 2009; Buschman and Kastner 2015; Fries 2015). Basal forebrain cholinergic input has been suggested to be involved in attentional modulation of cortical circuits (Hasselmo and McGaughy, 2004; Sarter et al., 2009; Schmitz and Duncan 2018; Thiele and Bellgrove 2018) but the cellular, circuit and synaptic mechanisms are complicated and in most cases remain to be elucidated.

Gamma band directional interactions were shown between $\mathrm{BF}$ and visual cortex during wake and sleep states (Nair et al. 2016) and BF stimulation enhances perception linked to LFP changes in visual cortical gamma (Goard and Dan 2009; Pinto et al. 2013). Furthermore, it has been shown that ACh release in the $\mathrm{mPFC}$ promotes gamma oscillations (Howe et al. 2017). Observations in the somatosensory cortex of mice, similarly, increased cholinergic input is linked to changes of high and low-frequency components of cortical LFPs (Eggermann et al. 2014; Kalmbach and Waters 2014).

We have shown that BF laser stimulation in ChAT-Cre rats induces significant coherence changes in high gamma in the OFC/V2 network at specific sites (Fig. 5, right column). BF neurons enhance gamma coherence between specific V2 and OFC sites that are likely to be interconnected. The fact that we did not observe significant coherence changes between all electrode pairs argues for the specificity of the main effect and suggests that it is not a methodological artifact.

There is good evidence that cortically projecting BF PV neurons are involved in cortical gamma band oscillations at $40 \mathrm{~Hz}$ (Yang et al. 2014; Kim et al. 2015; Dannenberg et al. 2015); however, this phenomenon may relate to $\mathrm{BF}$ cholinergic neurons exciting BF GABAergic projection neurons through cholinergic collaterals synapsing on PV cells (Zaborszky and Duque 2000).

\section{Cell assemblies in the BF}

Previous research (Lin et al. 2006) observed that a subset of BF neurons, called tonic firing neurons, bursted as transient $(160 \mathrm{~ms})$ ensembles, and their synchronization phase was tightly associated with prefrontal oscillation power. The responses of these putative non-cholinergic neurons correlated with the motivationally salient sensory cues that reliably predicted reinforcement, associated motor responses, and hedonic valences (reward or punishment), irrespective of their sensory modalities. However, BF bursting was absent when the same sensory cues were not motivationally salient (Lin and Nicolelis 2008). Studies by Nitz, Chiba and their colleagues emphasized that BF neurons form distinct 
task-phase ensembles within theta, beta, low and high gamma frequency windows (Tingley et al. 2018).

Defining short-latency functional interactions using a spike-jittering cross-correlation on a small subset of BF neurons suggests that the temporal interaction between two functional assemblies may be as little as $10-15 \mathrm{~ms}$ (Zaborszky and Gombkoto 2018). Behavior-dependent synchronization of BF neurons into cell assemblies in response to environmental or internal demands could be a mechanism by which widely separated cortical regions are quickly coordinated to facilitate or inhibit information flow between them as needed. This notion was predicted already from our early anatomical studies suggesting that "the BF is well-positioned anatomically to coordinate cortical oscillations among widely separated cortical regions and capable of binding these regions into larger functional networks" (Zaborszky 2002; Zaborszky et al. 2002). While studies by Nitz, Chiba and their colleagues suggest temporal coordination of BF neurons to form neuronal ensembles, our studies, by showing subtle differences in basalo-cortical coherence sites as assessed from the location of electrodes in the BF (Fig. 5d), indicate that cell ensembles in topographically different BF locations might be dynamically recruited during the various behavioral epochs.

\section{Cholinergic network in behaving rodents}

To our knowledge, cholinergic neurons in behaving rodents were identified first by Hangya et al. (Hangya et al. 2015) and Harrison et al. (Harrison et al. 2016). Cholinergic neurons responded to reward and punishment, similarly to unidentified BF neurons in primates by Monosov and his colleagues (Monosov et al. 2015; Zhang et al. 2019).

Our observations that the cholinergic neurons identified by optogenetic tagging (BF ID14-22, Fig. 3.a2) responded during decision-making or (BF ID 42-55; Fig. 3.a3) during reward-approach, and also that their STA modulated LFPs in specific OFC and V2 sites (Fig. 6, Table 1) suggest BF cholinergic modulation of specific cortical circuits. Nicotinic and muscarinic receptors are involved in specific modulation of gamma power in the prefrontal cortex (Kalmbach and Waters 2014). Due to the differential distribution of muscarinic and nicotinic receptors on pyramidal and various interneurons (Muñoz and Rudy 2014; Verhoog et al. 2016), the existence of two types of cholinergic cells in the BF of mice (Unal et al. 2012; Bloem et al. 2014), and the relative paucity of identified cholinergic neurons in our study, further research is necessary to find out which microcircuits are affected (Hasselmo and Cekic 1996; Xiang et al. 1998).

\section{Conjoint modulation of functional connectivity between visual and orbitofrontal cortex}

Orbital areas have reciprocal connections with V2, as shown using classical anterograde and retrograde tracers (Vogt and Miller 1983; Miller and Vogt 1984; Sanderson et al. 1991; Paperna and Malach 1991; Van Eden et al. 1992; Reep et al. 1996). The OFC has been suggested to be involved in many functions, including response inhibition, flexible representation of stimulus-outcome association, value coding, prediction error, coding of reward probability, and emotional appraisal; implying that OFC neurons play a major role of valuation and decision making. Neurons in the OFC respond both to primary reinforcers, as well as cues that predict rewards across multiple sensory domains, including visual, gustatory, somatosensory, olfactory, and auditory (Rolls and Deco 2006; Schoenbaum et al. 2009; Mainen and Kepecs 2009; O'Neill and Schultz 2010; Burke and Tobler 2011; Passingham and Wise 2012; McGinty et al. 2016; Sharpe and Schoenbaum 2016; Wikenheiser and Schoenbaum 2016; Izquierdo 2017).

In the OFC, we detected neurons that responded differently to visual cues (OFC neuron \#61-97, see Supplementary Fig. S3c) in the same region, where cells were encountered that responded while approaching the reward. In the OFC and V2 during decision making, and in $\mathrm{V} 2$ also during cue presentation, the gamma coherence increased; furthermore, the cholinergic STA in both cortical areas show spatially increased gamma power, thus it is likely that cholinergic neurons are involved in modulating gamma coherence between OFC/V2 regions during the decision to approach the reward.

\section{Concluding remarks}

The electrophysiological data suggest that cholinergic neurons together with other BF neurons participate in spatio-temporal coordination of cortical activity. This coordination may entail the exchange of information between specific interconnected cortical regions, which might underlie particular aspects of cognitive functions. This suggestion is in line with experiments showing coordinated ACh release in PFC and hippocampus (Teles-Grilo Ruivo et al. 2017). Acetylcholine can rapidly modulate the activity of specific circuits in the cortex (Sarter and Lustig, 2020). Although we focused on BF coordination of cortical ACh release, other mechanisms, including cortico-cortical, thalamo-cortical, and intrinsic connections between specific BF neurons may also play a role. 
These data and past anatomical studies (Li et al. 2017; Záborszky et al. 2018) suggest an emerging organization of the cholinergic system, in which functional cell assemblies may be dynamically recruited in correlation with behavior demands, through specific projections between distinct $\mathrm{BF}$ and cortical sites.

Supplementary Information The online version contains supplementary material available at https://doi.org/10.1007/s00429-021-02290-z.

Acknowledgements We thank Dr. Dave Sullivan and Dr. Drew Hadley for reading an earlier version of this manuscript.

Author contributions PG designed and conducted all the experiments. PG analyzed the data. MG, and CC were responsible for Nissl and antibody processing of the brains. PV comments were used for statistical analysis. LZ and PG wrote the manuscript.

Funding Funding was provided by NIH/NINDS Grant R01NS023945 and RF123945-28 to LZ and Rosztoczy Foundation Scholarship to PG.

Data availability All raw data are available from the authors.

\section{Declarations}

Conflict of interest The authors declare no competing interests.

Ethical standards Detailed protocols were approved by the Rutgers University Institutional Animal Care and Use Committee.

Open Access This article is licensed under a Creative Commons Attribution 4.0 International License, which permits use, sharing, adaptation, distribution and reproduction in any medium or format, as long as you give appropriate credit to the original author(s) and the source, provide a link to the Creative Commons licence, and indicate if changes were made. The images or other third party material in this article are included in the article's Creative Commons licence, unless indicated otherwise in a credit line to the material. If material is not included in the article's Creative Commons licence and your intended use is not permitted by statutory regulation or exceeds the permitted use, you will need to obtain permission directly from the copyright holder. To view a copy of this licence, visit http://creativecommons.org/licenses/by/4.0/.

\section{References}

Allen (2011) ALLEN Mouse Brain Connectivity Atlas. https://www. allenbrainatlas.org

Ballinger EC, Ananth M, Talmage DA, Role LW (2016) Basal forebrain cholinergic circuits and signaling in cognition and cognitive decline. Neuron 91:1199-1218. https://doi.org/10.1016/j.neuron. 2016.09.006

Barthó P, Hirase H, Monconduit L et al (2004) Characterization of neocortical principal cells and interneurons by network interactions and extracellular features. J Neurophysiol 92:600-608. https://doi. org/10.1152/jn.01170.2003

Bloem B, Schoppink L, Rotaru DC et al (2014) Topographic mapping between basal forebrain cholinergic neurons and the medial prefrontal cortex in mice. J Neurosci 34:16234-16246. https://doi. org/10.1523/JNEUROSCI.3011-14.201434/49/16234

Burke CJ, Tobler PN (2011) Coding of reward probability and risk by single neurons in animals. Front Neurosci 5:121. https://doi.org/ 10.3389/fnins.2011.00121

Buschman TJ, Kastner S (2015) From behavior to neural dynamics: an integrated theory of attention. Neuron 88:127-144. https://doi.org/ 10.1016/j.neuron.2015.09.017

Cardin JA, Carlén M, Meletis K et al (2009) Driving fast-spiking cells induces gamma rhythm and controls sensory responses. Nature 459:663-667. https://doi.org/10.1038/nature08002

Chavez C, Zaborszky L (2017) Basal forebrain cholinergic-auditory cortical network: primary versus nonprimary auditory cortical areas. Cereb Cortex 27:2335-2347. https://doi.org/10.1093/cercor/bhw091

Conner JM, Culberson A, Packowski C et al (2003) Lesions of the basal forebrain cholinergic system impair task acquisition and abolish cortical plasticity associated with motor skill learning. Neuron 38:819-829. https://doi.org/10.1016/S0896-6273(03)00288-5

Conner JM, Kulczycki M, Tuszynski MH (2010) Unique contributions of distinct cholinergic projections to motor cortical plasticity and learning. Cereb Cortex 20:2739-2748. https://doi.org/10.1093/ cercor/bhq022

Dannenberg H, Pabst M, Braganza O et al (2015) Synergy of direct and indirect cholinergic septo-hippocampal pathways coordinates firing in hippocampal networks. J Neurosci 35:8394-8410. https:// doi.org/10.1523/JNEUROSCI.4460-14.2015

Eggermann E, Kremer Y, Crochet S, Petersen CCH (2014) Cholinergic signals in mouse barrel cortex during active whisker sensing. Cell Rep 9:1654-1661. https://doi.org/10.1016/j.celrep.2014.11.005

Fries P (2015) Rhythms for cognition: communication through coherence. Neuron 88:220-235. https://doi.org/10.1016/j.neuron.2015. 09.034

Fujisawa S, Amarasingham A, Harrison MT, Buzsáki G (2008) Behavior-dependent short-term assembly dynamics in the medial prefrontal cortex. Nat Neurosci 11:823-833. https://doi.org/10.1038/ nn. 2134

Gielow MR, Zaborszky L (2017) The Input-output relationship of the cholinergic basal forebrain. Cell Rep 18:1817-1830. https://doi. org/10.1016/j.celrep.2017.01.060

Goard M, Dan Y (2009) Basal forebrain activation enhances cortical coding of natural scenes. Nat Neurosci 12:1444-1449. https://doi. org/10.1038/nn.2402

Gregoriou GG, Gotts SJ, Zhou H, Desimone R (2009) High-frequency, long-range coupling between prefrontal and visual cortex during attention. Science 324:1207-1210. https://doi.org/10.1126/scien ce. 1171402

Gritti I, Manns ID, Mainville L, Jones BE (2003) Parvalbumin, calbindin, or calretinin in cortically projecting and GABAergic, cholinergic, or glutamatergic basal forebrain neurons of the rat. $\mathrm{J}$ Comp Neurol 458:11-31. https://doi.org/10.1002/cne.10505

Hangya B, Ranade SP, Lorenc M, Kepecs A (2015) Central cholinergic neurons are rapidly recruited by reinforcement feedback. Cell 162:1155-1168. https://doi.org/10.1016/j.cell.2015.07.057

Harrison TC, Pinto L, Brock JR, Dan Y (2016) Calcium imaging of basal forebrain activity during innate and learned behaviors. Front Neural Circuits 10:1-12. https://doi.org/10.3389/fncir.2016.00036

Hasselmo ME (2006) The role of acetylcholine in learning and memory. Curr Opin Neurobiol 16:710-715. https://doi.org/10.1016/j. conb.2006.09.002

Hasselmo ME, Cekic M (1996) Suppression of synaptic transmission may allow combination of associative feedback and self-organizing feedforward connections in the neocortex. Behav Brain Res 79:153-161 
Hasselmo ME, McGaughy J (2004) High acetylcholine levels set circuit dynamics for attention and encoding and low acetylcholine levels set dynamics for consolidation. Prog Brain Res 145:207-231

Howe WM, Gritton HJ, Lusk NA et al (2017) Acetylcholine release in prefrontal cortex promotes gamma oscillations and theta-gamma coupling during cue detection. J Neurosci 37:3215-3230. https:// doi.org/10.1523/JNEUROSCI.2737-16.2017

Huppé-Gourgues F, Jegouic K, Vaucher E (2018) Topographic organization of cholinergic innervation from the basal forebrain to the visual cortex in the rat. Front Neural Circuits 12:1-10. https://doi. org/10.3389/fncir.2018.00019

Izquierdo A (2017) Functional heterogeneity within rat orbitofrontal cortex in reward learning and decision making. J Neurosci 37:10529. https://doi.org/10.1523/JNEUROSCI.1678-17.2017

Kalmbach A, Waters J (2014) Modulation of high- and low-frequency components of the cortical local field potential via nicotinic and muscarinic acetylcholine receptors in anesthetized mice. J Neurophysiol 111:258-272. https://doi.org/10.1152/jn.00244.2013

Kim T, Thankachan S, McKenna JT et al (2015) Cortically projecting basal forebrain parvalbumin neurons regulate cortical gamma band oscillations. Proc Natl Acad Sci 112:201413625. https://doi. org/10.1073/pnas.1413625112

Kim H, Ährlund-Richter S, Wang X et al (2016) Prefrontal parvalbumin neurons in control of attention. Cell 164:208-218. https://doi. org/10.1016/j.cell.2015.11.038

Lakatos P, Karmos G, Mehta AD et al (2008) Entrainment of neuronal oscillations as a mechanism of attentional selection. Science 320:110-113. https://doi.org/10.1126/science.1154735

Li X, Yu B, Sun Q et al (2017) Generation of a whole-brain atlas for the cholinergic system and mesoscopic projectome analysis of basal forebrain cholinergic neurons. Proc Natl Acad Sci. https:// doi.org/10.1073/pnas.1703601115

Lin SC, Nicolelis MAL (2008) Neuronal ensemble bursting in the basal forebrain encodes salience irrespective of valence. Neuron 59:138-149. https://doi.org/10.1016/j.neuron.2008.04.031

Lin S-C, Gervasoni D, Nicolelis MAL (2006) Fast modulation of prefrontal cortex activity by basal forebrain noncholinergic neuronal ensembles. J Neurophysiol 96:3209-3219. https://doi.org/ 10.1152/jn.00524.2006

Maguire LP, Szilagyi S, Scholten RE (2004) High performance laser shutter using a hard disk drive voice-coil actuator. Rev Sci Instrum 75:3077-3079. https://doi.org/10.1063/1.1786331

Mainen ZF, Kepecs A (2009) Neural representation of behavioral outcomes in the orbitofrontal cortex. Curr Opin Neurobiol 19:84-91. https://doi.org/10.1016/j.conb.2009.03.010

McGinty VB, Rangel A, Newsome WT (2016) Orbitofrontal cortex value signals depend on fixation location during free viewing. Neuron 90:1299-1311. https://doi.org/10.1016/j.neuron.2016. 04.045

Miller MW, Vogt BA (1984) Direct connections of rat visual cortex with sensory, motor, and association cortices. J Comp Neurol 226:184-202. https://doi.org/10.1002/cne.902260204

Minces VH, Alexander AS, Datlow M et al (2013) The role of visual cortex acetylcholine in learning to discriminate temporally modulated visual stimuli. Front Behav Neurosci 7:16. https:// doi.org/10.3389/fnbeh.2013.00016

Monosov IE, Leopold DA, Hikosaka O (2015) Neurons in the primate medial basal forebrain signal combined information about reward uncertainty, value, and punishment anticipation. J Neurosci 35:7443-7459. https://doi.org/10.1523/JNEUROSCI. 0051-15.2015

Muñoz W, Rudy B (2014) Spatiotemporal specificity in cholinergic control of neocortical function. Curr Opin Neurobiol 26:149-160

Nair J, Klaassen AL, Poirot J et al (2016) Gamma band directional interactions between basal forebrain and visual cortex during wake and sleep states. J Physiol Paris 110:19-28. https://doi. org/10.1016/j.jphysparis.2016.11.011

O'Neill M, Schultz W (2010) Coding of reward risk by orbitofrontal neurons is mostly distinct from coding of reward value. Neuron 68:789-800. https://doi.org/10.1016/j.neuron.2010.09.031

Paperna T, Malach R (1991) Patterns of sensory intermodality relationships in the cerebral cortex of the rat. J Comp Neurol 308:432-456. https://doi.org/10.1002/cne.903080310

Parikh V, Kozak R, Martinez V, Sarter M (2007) Prefrontal acetylcholine release controls cue detection on multiple timescales. Neuron 56:141-154. https://doi.org/10.1016/j.neuron.2007.08. 025

Passingham RE, Wise SP (2012) The neurobiology of the prefrontal cortex. Oxford University Press, Oxford

Paxinos G, Watson C (2007) The rat brain in stereotaxic coordinates, 6th edn. Elsevier Academic Press, Amsterdam

Pinto L, Goard MJ, Estandian D et al (2013) Fast modulation of visual perception by basal forebrain cholinergic neurons. Nat Neurosci 16:1857-1863. https://doi.org/10.1038/nn.3552

Prado VF, Janickova H, Al-Onaizi MA, Prado MAM (2017) Cholinergic circuits in cognitive flexibility. Neuroscience 345:130-141. https://doi.org/10.1016/j.neuroscience.2016.09.013

Reep RL, Corwin JV, King V (1996) Neuronal connections of orbital cortex in rats: topography of cortical and thalamic afferents. Exp Brain Res 111:215-232. https://doi.org/10.1007/BF00227299

Rolls ET, Deco G (2006) Attention in natural scenes: neurophysiological and computational bases. Neural Netw 19:1383-1394. https:// doi.org/10.1016/j.neunet.2006.08.007

Rossant C, Kadir SN, Goodman DFM et al (2016) Spike sorting for large, dense electrode arrays. Nat Neurosci 19:634-641. https:// doi.org/10.1038/nn.4268

Royer S, Zemelman BV, Barbic M et al (2010) Multi-array silicon probes with integrated optical fibers: light-assisted perturbation and recording of local neural circuits in the behaving animal. Eur J Neurosci 31:2279-2291. https://doi.org/10.1111/j.1460-9568. 2010.07250.x

Sanderson KJ, Dreher B, Gayer N (1991) Prosencephalic connections of striate and extrastriate areas of rat visual cortex. Exp Brain Res 85:324-334

Saper CB (1987) Diffuse cortical projection systems: anatomical organization and role in cortical function. Handb Physiol Nerv Syst Part 15:169-210. https://doi.org/10.1002/cphy.cp010506

Sarter M (2007) Cholinergic control of attention to cues guiding established performance versus learning: theoretical comment on Maddux, Kerfoot, Chatterjee, and Holland (2007). Behav Neurosci 121:233-235. https://doi.org/10.1037/0735-7044.121.1.233

Sarter M, Lustig C (2020) Forebrain cholinergic signaling: wired and phasic, not tonic, and causing behavior. J Neurosci 40:712-719

Sarter M, Parikh V, Howe WM (2009) Phasic acetylcholine release and the volume transmission hypothesis: time to move on. Nat Rev Neurosci 10:383-390

Schmitz TW, Duncan J (2018) Normalization and the cholinergic microcircuit: a unified basis for attention. Trends Cogn Sci 22:422-437. https://doi.org/10.1016/j.tics.2018.02.011

Schoenbaum G, Roesch MR, Stalnaker TA, Takahashi YK (2009) A new perspective on the role of the orbitofrontal cortex in adaptive behaviour. Nat Rev Neurosci. https://doi.org/10.1038/nrn2753

Senzai Y, Buzsáki G (2017) Physiological properties and behavioral correlates of hippocampal granule cells and mossy cells. Neuron 93:691-704.e5. https://doi.org/10.1016/j.neuron.2016.12.011

Sharpe MJ, Schoenbaum G (2016) Back to basics: making predictions in the orbitofrontal-amygdala circuit. Neurobiol Learn Mem 131:201-206. https://doi.org/10.1016/j.nlm.2016.04.009

Stark E, Abeles M (2009) Unbiased estimation of precise temporal correlations between spike trains. J Neurosci Methods 179:90-100. https://doi.org/10.1016/j.jneumeth.2008.12.029 
Teles-Grilo Ruivo LM, Baker KL, Conway MW et al (2017) Coordinated acetylcholine release in prefrontal cortex and hippocampus is associated with arousal and reward on distinct timescales. Cell Rep 18:905-917. https://doi.org/10.1016/j.celrep.2016.12.085

Thiele A, Bellgrove MA (2018) Neuromodulation of attention. Neuron 97:769-785. https://doi.org/10.1016/j.neuron.2018.01.008

Tingley D, Alexander AS, Kolbu S et al (2014) Task-phase-specific dynamics of basal forebrain neuronal ensembles. Front Syst Neurosci 8:174. https://doi.org/10.3389/fnsys.2014.00174

Tingley D, Alexander AS, Quinn LK et al (2015) Cell assemblies of the basal forebrain. J Neurosci 35:2992-3000. https://doi.org/10. 1523/JNEUROSCI.4432-14.2015

Tingley D, Alexander AS, Quinn LK et al (2018) Multiplexed oscillations and phase rate coding in the basal forebrain. Sci Adv 4:eaar3230. https://doi.org/10.1126/sciadv.aar3230

Unal CT, Golowasch JP, Zaborszky L (2012) Adult mouse basal forebrain harbors two distinct cholinergic populations defined by their electrophysiology. Front Behav Neurosci. https://doi.org/10.3389/ fnbeh.2012.00021

Van Eden CG, Lamme VAF, Uylings HBM (1992) Heterotopic cortical afferents to the medial prefrontal cortex in the rat. A combined retrograde and anterograde tracer study. Eur J Neurosci 4:77-97

Verhoog MB, Obermayer J, Kortleven CA et al (2016) Layer-specific cholinergic control of human and mouse cortical synaptic plasticity. Nat Commun 7:12826. https://doi.org/10.1038/ncomms12826

Vogt BA, Miller MW (1983) Cortical connections between rat cingulate cortex and visual, motor, and postsubicular cortices. J Comp Neurol 216:192-210. https://doi.org/10.1002/cne.902160207

Wikenheiser AM, Schoenbaum G (2016) Over the river, through the woods: cognitive maps in the hippocampus and orbitofrontal cortex. Nat Rev Neurosci 17:513-523. https://doi.org/10.1038/nrn. 2016.56

Witten IB, Steinberg EE, Lee SY et al (2011) Recombinase-driver rat lines: tools, techniques, and optogenetic application to dopaminemediated reinforcement. Neuron 72:721-733. https://doi.org/10. 1016/j.neuron.2011.10.028

Womelsdorf T, Schoffelen J-M, Oostenveld R et al (2007) Modulation of neuronal interactions through neuronal synchronization. Science 316:1609-1612. https://doi.org/10.1126/science.1139597

Xiang Z, Huguenard JR, Prince DA (1998) Cholinergic switching within neocortical inhibitory networks. Science 281:985-988. https://doi.org/10.1126/science.281.5379.985
Yague JG, Tsunematsu T, Sakata S (2017) Distinct temporal coordination of spontaneous population activity between basal forebrain and auditory cortex. Front Neural Circuits 11:64. https://doi.org/ 10.3389/fncir.2017.00064

Yang C, McKenna JT, Zant JC et al (2014) Cholinergic neurons excite cortically projecting basal forebrain GABAergic neurons. J Neurosci 34:2832-2844. https://doi.org/10.1523/JNEUROSCI.3235-13. 2014

Zaborszky L (2002) The modular organization of brain systems. Basal forebrain: the last frontier. Prog Brain Res 136:359-372

Zaborszky L, Duque A (2000) Local synaptic connections of basal forebrain neurons. Behav Brain Res 115:143-158

Zaborszky L, Gombkoto P (2018) The Cholinergic multicompartmental basal forebrain microcircuit. In: Shepherd G, Grillner S (eds) Handbook of brain microcircuits, 2nd edn. Oxford University Press, New York, pp 163-183

Zaborszky L, Csordas A, Buhl DL et al (2002) Computational anatomical analysis of the basal forebrain corticopetal system. In: Ascoli $\mathrm{G}$ (ed) Computational neuroanatomy. Principles and methods, Humana Press, Totowa, pp 171-197. https://doi.org/10.1007/ 978-1-59259-275-3_9

Zaborszky L, Csordas A, Mosca K et al (2015) Neurons in the basal forebrain project to the cortex in a complex topographic organization that reflects corticocortical connectivity patterns: an experimental study based on retrograde tracing and $3 \mathrm{D}$ reconstruction. Cereb Cortex 25:118-137. https://doi.org/10.1093/cercor/bht210

Záborszky L, Gombkoto P, Varsanyi P et al (2018) Specific basal forebrain-cortical cholinergic circuits coordinate cognitive operations. J Neurosci 38:9446-9458. https://doi.org/10.1523/JNEUROSCI. 1676-18.2018

Zhang K, Chen CD, Monosov IE (2019) Novelty, salience, and surprise timing are signaled by neurons in the basal forebrain. Curr Biol 29:134-142. https://doi.org/10.1016/j.cub.2018.11.012

Publisher's Note Springer Nature remains neutral with regard to jurisdictional claims in published maps and institutional affiliations. 